TRANSACTIONS OF THE

AMERICAN MATHEMATICAL SOCIETY

Volume 359, Number 4, April 2007, Pages 1889-1924

S 0002-9947(06)04112-2

Article electronically published on November 22, 2006

\title{
C*-ALGEBRAS ASSOCIATED WITH INTERVAL MAPS
}

\author{
VALENTIN DEACONU AND FRED SHULTZ
}

\begin{abstract}
For each piecewise monotonic map $\tau$ of $[0,1]$, we associate a pair of $\mathrm{C}^{*}$-algebras $F_{\tau}$ and $O_{\tau}$ and calculate their K-groups. The algebra $F_{\tau}$ is an AI-algebra. We characterize when $F_{\tau}$ and $O_{\tau}$ are simple. In those cases, $F_{\tau}$ has a unique trace, and $O_{\tau}$ is purely infinite with a unique KMS state. In the case that $\tau$ is Markov, these algebras include the Cuntz-Krieger algebras $O_{A}$, and the associated AF-algebras $F_{A}$. Other examples for which the K-groups are computed include tent maps, quadratic maps, multimodal maps, interval exchange maps, and $\beta$-transformations. For the case of interval exchange maps and of $\beta$-transformations, the $\mathrm{C}^{*}$-algebra $O_{\tau}$ coincides with the algebras defined by Putnam and Katayama-Matsumoto-Watatani, respectively.
\end{abstract}

\section{INTRODUCTION}

Motivation. There has been a fruitful interplay between $\mathrm{C}^{*}$-algebras and dynamical systems, which can be summarized by the diagram

$$
\text { dynamical system } \rightarrow \mathrm{C}^{*} \text {-algebra } \rightarrow K \text {-theoretic invariants. }
$$

A classic example is the pair of $\mathrm{C}^{*}$-algebras $F_{A}, O_{A}$ that Krieger and Cuntz [10] associated with a shift of finite type. They computed the K-groups of these algebras, and observed that $K_{0}\left(F_{A}\right)$ was the same as a dimension group that Krieger had earlier defined in dynamical terms [34. Krieger 35] had shown that this dimension group, with an associated automorphism, determined the matrix $A$ up to shift equivalence. Later it was shown that this dimension group and automorphism determines the two-sided shift up to eventual conjugacy; cf. 43]. Cuntz [9] showed that the K-groups of $O_{A}$ are exactly the Bowen-Franks invariants for flow equivalence. Not only have these algebras provided interesting invariants for the dynamical systems, but the favor has been returned, in the sense that these algebras have been an inspiration for many developments in $\mathrm{C}^{*}$-algebras.

Given a piecewise monotonic map $\tau$ of the unit interval, we will associate two $\mathrm{C}^{*}$-algebras $F_{\tau}, O_{\tau}$, and calculate their K-theory, thus getting invariants for $\tau$. The invariants are computable in terms of the original map and standard concepts from dynamical systems.

Received by the editors August 1, 2004 and, in revised form, June 11, 2005.

2000 Mathematics Subject Classification. Primary 46L80; Secondary 37E05.

Key words and phrases. Dimension group, interval map, piecewise monotonic, unimodal map, tent map, Markov map, $\beta$-shift, interval exchange map, $C^{*}$-algebra, Cuntz-Krieger algebra, Matsumoto algebra.

(C)2006 American Mathematical Society Reverts to public domain 28 years from publication 
Construction. As our notation indicates, our algebras $F_{\tau}$ and $O_{\tau}$ are generalizations of $F_{A}$ and $O_{A}$, respectively. They include the algebras $F_{A}$ and $O_{A}$ as special cases (Corollary 11.9), but also include simple $\mathrm{C}^{*}$-algebras that are not Cuntz-Krieger algebras (Examples 13.1, 13.2).

Before defining these algebras, we review the construction of two related algebras $C^{*}(R(X, \sigma))$ and $C^{*}(X, \sigma)$. Suppose that $X$ is a compact Hausdorff space, and $\sigma: X \rightarrow X$ is a local homeomorphism. Define an equivalence relation

$$
R(X, \sigma)=\left\{(x, y) \in X \times X \mid \sigma^{n} x=\sigma^{n} y \text { for some } n \geq 0\right\},
$$

with a suitable locally compact topology. For $f, g \in C_{c}(R(X, \sigma))$, define a convolution product by $(f * g)(x, y)=\sum_{z \sim x} f(x, z) g(z, y)$, and an involution $*$ by $f^{*}(x, y)=\overline{f(y, x)}$. Then $C_{c}(R(X, \sigma))$ becomes a *-algebra, which can be normed and completed to become a $\mathrm{C}^{*}$-algebra $C^{*}(R(X, \sigma))$. This is a special case of the construction of $\mathrm{C}^{*}$-algebras from locally compact groupoids, so one can make use of the extensive machinery developed by Renault [58]. The definition of $C^{*}(X, \sigma)$ is similar, except that $R(X, \sigma)$ is replaced by

$$
G(X, \sigma)=\left\{(x, n, y) \in X \times \mathbb{Z} \times X \mid \sigma^{j} x=\sigma^{k} y \text { and } n=k-j\right\} .
$$

This construction is due to Deaconu 12. It generalizes an example of Renault in [58, and is generalized further in [1, 59]. It is closely related to a construction of Arzumanian and Vershik [3] in a measure-theoretic context. If this construction is applied to the local homeomorphism given by a one-sided shift of finite type associated with a zero-one matrix $A$, then $C^{*}(R(X, \sigma)) \cong F_{A}$ and $C^{*}(X, \sigma) \cong O_{A}$; cf. [12, 59.

For the construction above to work, it is crucial that $\sigma$ be a local homeomorphism. Therefore, if $\tau: I \rightarrow I$ is piecewise monotonic, we associate with $\tau$ a local homeomorphism $\sigma: X \rightarrow X$, constructed by disconnecting the interval $I=[0,1]$ at points in the forward and backward orbit of the endpoints of intervals of monotonicity. This is a well-known technique for one-dimensional dynamical systems; cf. [30, 24, 62, 66. Then we define $F_{\tau}=C^{*}(R(X, \sigma))$ and $O_{\tau}=C^{*}(X, \sigma)$. (There are alternative, equivalent, ways to define $F_{\tau}$ and $O_{\tau}$. For example, $F_{\tau}$ can be defined directly as an inductive limit of interval algebras (cf. 63, Prop. 12.3] and [14, Thm. 8.1]), or $F_{\tau}$ and $O_{\tau}$ can be defined as the $\mathrm{C}^{*}$-algebras $\mathcal{F}_{E}$ and $\mathcal{O}_{E}$ defined by Pimsner [51, where $E$ is an appropriate Hilbert module. For details, see [13.)

If $\tau$ is surjective, from its construction, $F_{\tau}$ comes equipped with a canonical endomorphism $\Phi$, so we have a $\mathrm{C}^{*}$-dynamical system $\left(F_{\tau}, \Phi\right)$. The algebra $O_{\tau}$ is isomorphic to the crossed product $F_{\tau} \times_{\Phi} \mathbb{N}$, i.e., the universal $\mathrm{C}^{*}$-algebra containing a copy of $F_{\tau}$ and an isometry implementing $\Phi$.

Summary of results. We describe the $K$-groups of these algebras in dynamical terms, show that they determine the $\mathrm{C}^{*}$-algebras, and show that the properties of the $\mathrm{C}^{*}$-algebras are closely related to those of the dynamical system $\tau: I \rightarrow I$.

Theorem (Propositions 6.7, 6.10). $F_{\tau}$ is always an AI-algebra (Definition 6.9). It is an AF-algebra iff $\tau$ has no homtervals.

(For $\tau: I \rightarrow I$, an interval $J$ is a homterval if $\tau^{n}$ restricted to $J$ is a homeomorphism for all $n \geq 0$. The dynamical behavior of an interval map on a homterval is particularly simple, and there are no homtervals if, for example, $\tau$ is transitive. 
Background on homtervals can be found in Collet-Eckmann [ [8, §II.5] or de Melovan Strien [44, Lemma II.3.1].)

Theorem (Propositions 5.2, 5.3). If $\tau$ is surjective, then $F_{\tau}$ is simple iff $\tau$ is topologically exact (Definitions 2.6, 2.7), and $O_{\tau}$ is simple iff $\tau$ is transitive.

If $\left(X_{A}, \sigma_{A}\right)$ is a transitive one-sided shift of finite type, it is well known that $X_{A}$ decomposes into $p<\infty$ pieces permuted cyclically by $\sigma_{A}$, such that $\sigma_{A}^{p}$ is mixing on each piece. The same kind of decomposition is valid for transitive piecewise monotonic maps (cf. 64, Cor. 4.7]), and for the associated algebra $F_{\tau}$. However, interestingly, we have to exclude the case where $\tau$ is bijective (as can be seen from the map $x \mapsto x+\alpha \bmod 1$, with $\alpha$ irrational). We say $\tau$ is essentially injective if it is injective on the complement of a finite set of points, which is equivalent to the associated local homeomorphism $\sigma$ being a homeomorphism. Of course, a continuous transitive map of the interval cannot be essentially injective.

Theorem (Corollary 6.13). If $\tau$ is transitive and not essentially injective, then $F_{\tau}$ is a finite direct sum $A_{1} \oplus \cdots \oplus A_{n}$ of simple $A F$-algebras, each possessing a unique tracial state. The endomorphism $\Phi$ maps each $A_{i}$ to $A_{i-1} \bmod n$.

Theorem (Theorem 9.5). If $\tau$ is transitive and not essentially injective, then $O_{\tau}$ is separable, simple, purely infinite, and nuclear, and it is in the UCT class.

Under the hypotheses above, by the classification results of Phillips [50] and Kirchberg [33, it follows that $O_{\tau}$ is determined by its K-groups.

Since $F_{\tau}$ is an AI-algebra, then $K_{0}\left(F_{\tau}\right)$ is a dimension group, i.e., an inductive limit of ordered groups of the form $\mathbb{Z}^{n_{k}}$, and $K_{1}\left(F_{\tau}\right)=0$. If $\tau$ is surjective, the endomorphism $\Phi$ induces an automorphism $\Phi_{*}$ of $K_{0}\left(F_{\tau}\right)$. We then refer to $\left(K_{0}\left(F_{\tau}\right), K_{0}\left(F_{\tau}\right)^{+}, \Phi_{*}\right)$ as the dimension triple associated with $\tau$.

In 63, 64, a dimension triple $\left(D G(\tau), D G(\tau)^{+}, \mathcal{L}_{*}\right)$ is defined in dynamical terms and investigated for various families of interval maps. Here $\mathcal{L}: C(X, \mathbb{Z}) \rightarrow C(X, \mathbb{Z})$ is the transfer operator (Definition [7.2).

Theorem (Corollary 17.10). If $\tau$ is surjective, then

$$
\left(K_{0}\left(F_{\tau}\right), K_{0}\left(F_{\tau}\right)^{+}, \Phi_{*}\right) \cong\left(D G(\tau), D G(\tau)^{+}, \mathcal{L}_{*}\right) .
$$

Combining this description of $K_{0}$ with Paschke's exact sequence 49 for crossed products by endomorphisms allows us to describe the K-groups of $O_{\tau}$.

Theorem (Proposition 9.4). If $\tau$ is surjective,

$$
\begin{array}{ll}
K_{0}\left(O_{\tau}\right) \cong D G(\tau) / \operatorname{im}\left(\mathrm{id}-\mathcal{L}_{*}\right) & \cong C(X, \mathbb{Z}) / \operatorname{im}(\mathrm{id}-\mathcal{L}), \\
K_{1}\left(O_{\tau}\right) \cong \operatorname{ker}\left(\operatorname{id}-\mathcal{L}_{*}\right) & \cong \operatorname{ker}(\mathrm{id}-\mathcal{L}) .
\end{array}
$$

In the case when $\tau$ is transitive, we can also describe the traces on $F_{\tau}$ and the KMS states on $O_{\tau}$.

Theorem (Theorems 10.5, 10.7). If $\tau$ is transitive and not essentially injective, there is a unique tracial state on $F_{\tau}$ scaled by the canonical endomorphism $\Phi$. The trace is given by the unique scaled measure on I (Definition 10.1), and the scaling factor is $\exp h_{\tau}$, where $h_{\tau}$ is the topological entropy of $\tau$. Furthermore, $O_{\tau}$ has a unique $\beta-K M S$ state for $\beta=h_{\tau}$, and there is no other $\beta$-KMS state for $0 \leq \beta<\infty$.

After establishing the results described above, we apply them in sections 11 15 to particular families of maps, including unimodal maps, multimodal maps, interval 
exchange maps, and $\beta$-transformations. As can be seen from the results above, the K-groups of $F_{\tau}$ and $O_{\tau}$ can be recovered from the dynamically defined dimension triple $\left(D G(\tau), D G(\tau)^{+}, \mathcal{L}_{*}\right)$. By making use of the study of this dimension triple in [63, 64, we are able to give explicit descriptions of the K-groups in terms of the map $\tau$ for these families of maps.

Other $\mathrm{C}^{*}$-algebras associated with interval maps. Other authors have associated $\mathrm{C}^{*}$-algebras with particular families of interval maps that coincide with ours. For example, for interval exchange maps $\tau: I \rightarrow I$, Putnam [55, 56] associated a homeomorphism $\sigma: X \rightarrow X$, which is identical to the local homeomorphism we associated with $\tau$ viewed as a piecewise monotonic map. The crossed product algebra he analyzes is the same as our $O_{\tau}$.

Katayama-Matsumoto-Watatani [27] defined $\mathrm{C}^{*}$-algebras $F_{\beta}^{\infty}$ and $O_{\beta}$ for $\beta$ shifts. If $\tau$ is the associated $\beta$-transformation, for all $\beta, O_{\tau} \cong O_{\beta}$ (Corollary 15.3). When the orbit of 1 under $\tau$ is finite, $F_{\tau} \cong F_{\beta}^{\infty}$ (Corollary 15.3). If the orbit of 1 is infinite, the dimension groups $K_{0}\left(F_{\beta}^{\infty}\right)$ and $K_{0}\left(F_{\tau}\right)$ are isomorphic as groups, but it is unknown whether they are order isomorphic, and thus unknown whether $F_{\beta}^{\infty}$ and $F_{\tau}$ are isomorphic. The algebras $F_{\beta}^{\infty}$ and $O_{\beta}$ are defined in quite different ways than our $F_{\tau}$ and $O_{\tau}$; it is only through their K-groups that we are able to establish these isomorphisms.

Other authors have defined $\mathrm{C}^{*}$-algebras for particular families of interval maps in ways that give different $\mathrm{C}^{*}$-algebras from those we have defined. Deaconu and Muhly [13] extended the local homeomorphism approach to branched coverings, which, for example, includes the full tent map. The $\mathrm{C}^{*}$-algebra they associate with the full tent map is not simple; thus it is not the same as our $O_{\tau}$. Renault [59] investigated groupoids and the associated $\mathrm{C}^{*}$-algebras associated with partially defined local homeomorphisms, which includes branched coverings as a special case. Martins-Severino-Ramos [40] associated a zero-one matrix $A$ with quadratic maps whose kneading sequence is periodic, and then computed the K-groups of $O_{A}$ in terms of that kneading sequence. Since the kneading sequence can be periodic without the critical point being periodic, the algebra $O_{\tau}$ we associate with such maps is generally different than their $O_{A}$, though the computation of $K_{0}\left(O_{A}\right)$ in terms of the kneading sequence is quite similar. For maps on the interval that are open maps, Exel [20] has defined a $\mathrm{C}^{*}$-algebra, which turns out to be isomorphic to the Cuntz algebra $O_{\infty}$ for the tent map, while our $O_{\tau}$ is isomorphic to $O_{2}$. Kajiwara and Watatani [26] have associated a $\mathrm{C}^{*}$-algebra with the complex dynamical systems given by a polynomial, acting on either the whole Riemann sphere, the Julia set, or its complement. In a few cases (e.g. $f(z)=z^{2}-2$ ) the Julia set is an interval, so that $f$ on its Julia set can be viewed as an interval map. For this dynamical system the algebra of Kajiwara and Watatani is isomorphic to $O_{\infty}$, while the corresponding algebra $\mathrm{O}_{\tau}$ we define is isomorphic to $\mathrm{O}_{2}$.

Another approach that can be taken when $\tau:[0,1] \rightarrow[0,1]$ is continuous and surjective is to form the inverse limit $\left(X^{\infty}, \tau^{\infty}\right)$, which will be a homeomorphism, and then form the crossed product $C\left(X^{\infty}\right) \times_{\tau^{\infty}} \mathbb{Z}$. This is a special case of the approach of Brenken [5], who associates a Cuntz-Pimsner $\mathrm{C}^{*}$-algebra with closed relations (such as the graph of a function). Since the inverse limits will have fixed points, the crossed product algebras will not be simple, so this also generally gives different algebras than those we consider. 


\section{LOCAL HOMEOMORPHISMS ASSOCIATED WITH PIECEWISE MONOTONIC MAPS}

In this section we review a construction in 63, which to each piecewise monotonic map $\tau:[0,1] \rightarrow[0,1]$ associates a local homeomorphism $\sigma: X \rightarrow X$ on $X \subset \mathbb{R}$, where $X$ is constructed by disconnecting $[0,1]$ at certain points.

Definition 2.1. Let $I=[0,1]$. A map $\tau: I \rightarrow I$ is piecewise monotonic if there are points $0=a_{0}<a_{1}<\cdots<a_{n}=1$ such that $\left.\tau\right|_{\left(a_{i-1}, a_{i}\right)}$ is continuous and strictly monotonic for $1 \leq i \leq n$. We will assume the sequence $a_{0}, a_{1}, \ldots, a_{n}$ is chosen so that no interval $\left(a_{i-1}, a_{i}\right)$ is contained in a larger open interval on which $\tau$ is continuous and strictly monotonic. The sequence of points $0=a_{0}<a_{1}<\cdots<$ $a_{n}=1$ is the partition associated with $\tau$, and the intervals $\left\{\left(a_{i-1}, a_{i}\right) \mid 1 \leq i \leq n\right\}$ are called the intervals of monotonicity for $\tau$. Note that for $1 \leq i \leq n$, the map $\left.\tau\right|_{\left(a_{i-1}, a_{i}\right)}$ extends uniquely to a strictly monotonic continuous map $\tau_{i}:\left[a_{i-1}, a_{i}\right] \rightarrow$ $I$, which will be a homeomorphism onto its image.

If $\tau$ is not continuous, we will ignore the actual values of $\tau$ at the partition points, and instead view $\tau$ as being multivalued, with the values at $a_{i}$ (for $1 \leq i \leq n-1$ ) being the values given by left and right limits, i.e., the values of $\tau$ at $a_{i}$ are $\tau_{i}\left(a_{i}\right)$ and $\tau_{i+1}\left(a_{i}\right)$. We define a (possibly multivalued) function $\widehat{\tau}$ on $I$ by setting $\widehat{\tau}(x)$ to be the set of left and right limits of $\tau$ at $x$. At points where $\tau$ is continuous, $\widehat{\tau}(x)=\{\tau(x)\}$, and we identify $\widehat{\tau}(x)$ with $\tau(x)$. Thus for $A \subset I, \widehat{\tau}(A)=\bigcup_{i} \tau_{i}\left(A \cap\left[a_{i-1}, a_{i}\right]\right)$ and $\widehat{\tau}^{-1}(A)=\bigcup_{i}\left\{x \in\left[a_{i-1}, a_{i}\right] \mid \tau_{i}(x) \in A\right\}$.

If $x \in I$, the generalized orbit of $x$ is the smallest subset of $I$ containing $x$ and forward and backward invariant with respect to $\widehat{\tau}$. Let $I_{1}$ be the union of the generalized orbits of $a_{0}, a_{1}, \ldots, a_{n}$, and let $I_{0}=I \backslash I_{1}$. The set $I_{0}$ is dense in $I$, and both $I_{0}$ and $I_{1}$ are forward and backward invariant with respect to $\widehat{\tau}$.

Definition 2.2. Let $I=[0,1]$, and let $I_{0}, I_{1}$ be as above. The disconnection of $I$ at points in $I_{1}$ is the totally ordered set $X$ which consists of a copy of $I$ with the usual ordering, but with each point $x \in I_{1} \backslash\{0,1\}$ replaced by two points $x^{-}<x^{+}$. We equip $X$ with the order topology, and define the collapse map $\pi: X \rightarrow I$ by $\pi\left(x^{ \pm}\right)=x$ for $x \in I_{1}$, and $\pi(x)=x$ for $x \in I_{0}$. We write $X_{1}=\pi^{-1}\left(I_{1}\right)$ and $X_{0}=\pi^{-1}\left(I_{0}\right)=X \backslash X_{1}$.

Here $X$ will be homeomorphic to a compact subset of $\mathbb{R}$.

Proposition 2.3. Let $\tau: I \rightarrow I$ be piecewise monotonic, and $X$ as in Definition 2.2. Then

(1) $\pi$ is continuous, and $\left.\pi\right|_{X_{0}}$ is a homeomorphism from $X_{0}$ onto $I_{0}$.

(2) $X_{0}$ is dense in $X$.

Proof. [63, Prop. 2.2].

Notation. If $b_{1}, b_{2} \in I_{1}$ with $b_{1}<b_{2}$, then $I\left(b_{1}, b_{2}\right)$ is the order interval $\left[b_{1}^{+}, b_{2}^{-}\right]_{X}$. If $b_{1}>b_{2}$, then we define $I\left(b_{1}, b_{2}\right)=I\left(b_{2}, b_{1}\right)$, and if $b_{1}=b_{2}$, then we set $I\left(b_{1}, b_{2}\right)=\emptyset$. Each set $I\left(b_{1}, b_{2}\right)$ will be clopen in $X$, and every clopen subset of $X$ is a finite union of such sets [63, Prop. 2.2].

Proposition 2.4. Let $\tau: I \rightarrow I$ be a piecewise monotonic map, with associated partition $a_{0}<a_{1}<\cdots<a_{n}$, and let $(X, \pi)$ be as described in Definition 2.2 .

(1) There is a unique continuous map $\sigma: X \rightarrow X$ such that $\pi \circ \sigma=\tau \circ \pi$ on $X_{0}$. 
(2) The sets $X_{0}$ and $X_{1}=X \backslash X_{0}$ are forward and backward invariant with respect to $\sigma$.

(3) $\pi$ is a conjugacy from $\left.\sigma\right|_{X_{0}}$ onto $\left.\tau\right|_{I_{0}}$.

(4) The sets $J_{1}=I\left(a_{0}, a_{1}\right), \ldots, J_{n}=I\left(a_{n-1}, a_{n}\right)$ are a partition of $X$ into clopen sets such that for $1 \leq i \leq n, \pi\left(J_{i}\right)=\left[a_{i-1}, a_{i}\right]$, and $\sigma \mid J_{i}$ is a homeomorphism from $J_{i}$ onto $\sigma\left(J_{i}\right)$.

(5) For $1 \leq i \leq n, \pi \circ \sigma=\tau_{i} \circ \pi$ on $J_{i}$.

Proof. [63, Theorem 2.3].

Note that if $\tau$ is continuous, by (3) and the density of $I_{0}$ in $I$ and $X_{0}$ in $X, \pi$ will be a semi-conjugacy from $(X, \sigma)$ onto $(I, \tau)$. We will call $\sigma$ the local homeomorphism associated with $\tau$. The map $\sigma$ actually has a somewhat stronger property, which will play an important role in our dynamical description of $K_{0}\left(F_{\tau}\right)$ (Corollary (7.10).

Definition 2.5. Let $X$ be a compact metric space. A continuous map $\sigma: X \rightarrow X$ is a piecewise homeomorphism if $\sigma$ is open and there is a partition of $X$ into clopen sets, each mapped homeomorphically onto its image.

If $\tau: I \rightarrow I$ is piecewise monotonic and $\sigma: X \rightarrow X$ is the associated local homeomorphism, then $\sigma$ will be a piecewise homeomorphism by Proposition 2.4(4).

If $X$ is any compact metric space and is zero dimensional, then any local homeomorphism $\sigma: X \rightarrow X$ is a piecewise homeomorphism. (For each point $x$, pick a clopen neighborhood on which $\sigma$ is injective, then select a finite cover of such clopen sets, and finally refine the cover to form a partition.)

Property (3) of Proposition 2.4 can be used to show that $\sigma$ and $\tau$ share many properties. Before being more explicit, we review some terminology.

Definition 2.6. If $X$ is any topological space and $f: X \rightarrow X$ is a continuous map, then $f$ is transitive if for each pair $U, V$ of non-empty open sets, there exists $n \geq 0$ such that $f^{n}(U) \cap V \neq \emptyset$. We say $f$ is strongly transitive if for every non-empty open set $U$, there exists $n$ such that $\bigcup_{k=0}^{n} f^{k}(U)=X$. The map $f$ is (topologically) mixing if for every pair $U, V$ of non-empty open sets, there exists $N$ such that for all $n \geq N, f^{n}(U) \cap V \neq \emptyset$, and $f$ is topologically exact if for every non-empty open set $U$, there exists $n$ such that $f^{n}(U)=X$.

Definition 2.7. If $\tau: I \rightarrow I$ is piecewise monotonic, we view $\tau$ as undefined at the set $C$ of endpoints of intervals of monotonicity, and say $\tau$ is transitive if for each pair $U, V$ of non-empty open sets, there exists $n \geq 0$ such that $\tau^{n}(U) \cap V \neq \emptyset$, and is topologically mixing if for each pair $U, V$ of non-empty open sets, there exists $N \geq 0$ such that for all $n \geq N, \tau^{n}(U) \cap V \neq \emptyset$. We say $\tau$ is strongly transitive if for every non-empty open set $U$, there exists $n$ such that $\bigcup_{k=0}^{n} \widehat{\tau}^{k}(U)=I$. The map $\tau$ is topologically exact if for every non-empty open set $U$, there exists $n$ such that $\widehat{\tau}^{n}(U)=I$. (If $\tau$ is continuous and piecewise monotonic, these definitions are consistent with those in Definition 2.6.)

Clearly every strongly transitive map is transitive, and every topologically exact map is topologically mixing. The converse implications do not hold, as can be seen by considering the two-sided $n$-shift. However, every continuous piecewise monotonic topologically mixing map is topologically exact; cf. [54 Thm. 2.5] and [6. Thm. 45]. See also [64, Prop. 4.9] for an additional result relating mixing and exactness. 
Proposition 2.8. Let $\tau: I \rightarrow I$ be piecewise monotonic and $\sigma: X \rightarrow X$ the associated local homeomorphism.

(1) $\sigma$ is surjective iff $\tau$ is surjective.

(2) $\sigma$ is strongly transitive iff $\tau$ is strongly transitive iff $\tau$ is transitive iff $\sigma$ is transitive.

(3) $\sigma$ is topologically exact iff $\tau$ is topologically exact.

(4) The topological entropy of $\sigma$ is equal to the topological entropy of $\tau$.

Proof. 63, Lemma 4.2, Prop. 2.9, Lemma 5.2] and [64, Prop. 2.8].

\section{C*-AlgEBRAS ASSOCIATED With LOCAL HOMEOMORPHISMS}

Let $X$ be a compact metric space, and $\sigma: X \rightarrow X$ a local homeomorphism, i.e., a continuous open map such that each point admits an open neighborhood on which $\sigma$ is injective. In this section we will review the construction of two $\mathrm{C}^{*}$-algebras associated with $\sigma$. (While this construction is well known, we will need to refer to some of the details later.)

We first describe two locally compact groupoids associated with $\sigma$. (The standard reference for locally compact groupoids and their associated $\mathrm{C}^{*}$-algebras is the book of Renault [58]). These groupoids were first described in [58, for the shift map $\sigma$ on the space $\Sigma_{n}$ of sequences $\{1,2, \ldots, n\}^{\mathbb{N}}$, for $p$-fold covering maps in [12, for surjective local homeomorphisms in [1] and in 2], and for general local homeomorphisms in [59]. Similar constructions in a measure-theoretic context were introduced in [3].

For each $n \in \mathbb{N}$, let

$$
R_{n}=\left\{(x, y) \in X \times X \mid \sigma^{n} x=\sigma^{n} y\right\}
$$

and $R(X, \sigma)=\bigcup_{n} R_{n}$. Give each $R_{n}$ the product topology from $X \times X$, and give $R(X, \sigma)$ the inductive limit topology. We write $x \sim y$ when $(x, y) \in R(X, \sigma)$, and define a product and involution on $C_{c}(R(X, \sigma))$ by

$$
(f \star g)(x, y)=\sum_{z \sim x} f(x, z) g(z, y), \quad f^{*}(x, y)=\overline{f(y, x)} .
$$

Each $R_{n}$ is a compact open subset of $R(X, \sigma)$, so we can identify $C\left(R_{n}\right)$ with the functions in $C_{c}(R(X, \sigma))$ whose support is contained in $R_{n}$. Thus $C_{c}(R(X, \sigma))=$ $\bigcup_{n=0}^{\infty} C\left(R_{n}\right)$. Each subspace $C\left(R_{n}\right)$ will be a ${ }^{*}$-subalgebra of $C_{c}(R(X, \sigma))$. By [58, Prop. II.4.2] there is a norm (necessarily unique) making $C\left(R_{n}\right)$ a $\mathrm{C}^{*}$-algebra, which we denote by $C^{*}\left(R_{n}\right)$. Since $C_{c}(R(X, \sigma))$ is the union of the subalgebras $C^{*}\left(R_{n}\right)$, there is a unique norm on $C_{c}(R(X, \sigma))$ satisfying the $\mathrm{C}^{*}$ norm axioms, and the completion is a $\mathrm{C}^{*}$-algebra that we denote $C^{*}(R(X, \sigma))$. (By the uniqueness of the $\mathrm{C}^{*}$ norm on $C_{c}(R(X, \sigma))$, the full and reduced $\mathrm{C}^{*}$-algebras of $R(X, \sigma)$ coincide; cf. [59, proof of Prop. 2.4].) From the discussion above, $C^{*}(R(X, \sigma))$ is the inductive limit of the algebras $C^{*}\left(R_{n}\right)$; cf. [60, Cor. 2.2].

Now we define a second groupoid

$$
G(X, \sigma)=\left\{(x, n, y) \in X \times \mathbb{Z} \times X \mid \exists k, l, \text { with } \sigma^{k} x=\sigma^{l} y \text { and } n=k-l\right\},
$$

with the product $(x, m, y)(y, n, z)=(x, m+n, z)$, inverse $(x, n, y)^{-1}=(y,-n, x)$, and with the topology given by the basis of open sets

$$
N(U, V, k, l)=\left\{(x, k-l, y) \in U \times \mathbb{Z} \times V \mid \sigma^{k} x=\sigma^{l} y\right\},
$$


where $\sigma^{k}$ and $\sigma^{l}$ are injective on the open sets $U$ and $V$. Then we can define a *-algebra $C_{c}(G(X, \sigma))$ by a convolution product, and for a suitable norm the completion is a $\mathrm{C}^{*}$-algebra, denoted by $C^{*}(G(X, \sigma))$ or by $C^{*}(X, \sigma)$. (The reduced and full $\mathrm{C}^{*}$-algebras associated with $G(X, \sigma)$ coincide; cf. [59, Prop. 2.4]. We remark that if $\sigma$ is a homeomorphism, then the map $(x, m) \mapsto\left(x, m, \sigma^{m} x\right)$ is a homeomorphism and algebraic isomorphism from the groupoid $X \times_{\phi} \mathbb{Z}$ associated with the transformation group $\mathbb{Z}$ acting on $X$, onto $G(X, \sigma)$. In that context, it follows that $C^{*}(X, \sigma) \cong C(X) \times_{\psi} \mathbb{Z}$, where $\psi(f)=f \circ \sigma^{-1}$; cf. [58.

There is a close relationship between the two $\mathrm{C}^{*}$-algebras we have just defined. Suppose that $\sigma$ is surjective, and let $A=C^{*}(R(X, \sigma))$. Then the map $\Phi: C_{c}(R(X, \sigma)) \rightarrow C_{c}(R(X, \sigma))$ given by

$$
\Phi(f)(x, y)=\frac{1}{\sqrt{p(\sigma(x)) p(\sigma(y))}} f(\sigma(x), \sigma(y)),
$$

where $p(z)$ is the cardinality of $\sigma^{-1}(z)$, extends to a ${ }^{*}$-endomorphism from $A$ into $A$. The image of $\Phi$ is $q A q$, where $q=\Phi(1)$. If $\sigma$ is not injective, then $q \neq 1$. Thus, in the terminology of Paschke [48], $\Phi$ will be a proper corner endomorphism. Therefore we can form the crossed product $A \times_{\Phi} \mathbb{N}$ (the universal $\mathrm{C}^{*}$-algebra generated by a copy of $A$ and a non-unitary isometry implementing $\Phi)$, and $C^{*}(X, \sigma)$ will be isomorphic to $A \times_{\Phi} \mathbb{N}$; cf. [12] and [1].

Many maps of the interval that are of interest are not surjective, e.g. the members of the logistic family $L_{k}$ given by $L_{k}(x)=k x(1-x)$. However, these maps are often eventually surjective. We say a map $\sigma: X \rightarrow X$ is eventually surjective if there exists an integer $n \geq 0$ such that $\sigma^{n+1}(X)=\sigma^{n}(X)$. In that case we call $\sigma^{n}(X)$ the eventual range, and $\sigma$ restricted to its eventual range is surjective. For example, if $2 \leq k<4$, the map $L_{k}$ is not surjective but is eventually surjective with eventual range being the interval $\left[0, L_{k}(1 / 2)\right]$.

If $X$ is a compact Hausdorff space and $\sigma: X \rightarrow X$ is an eventually surjective local homeomorphism, with eventual range $Y$, then the algebra $C^{*}(R(X, \sigma))$ is strongly Morita equivalent to $C^{*}\left(R\left(Y,\left.\sigma\right|_{Y}\right)\right)$, and $C^{*}(X, \sigma)$ is strongly Morita equivalent to $C^{*}\left(Y,\left.\sigma\right|_{Y}\right)$. This follows from the fact that each $R(X, \sigma)$ equivalence class meets $Y$; cf. [41, Ex. 2.7 and Thm. 2.8]. Thus for our purposes there is not much lost by restricting consideration to surjective maps, and we will do that whenever it is convenient.

\section{Simplicity of $C^{*}(R(X, \sigma))$ And $C^{*}(X, \sigma)$}

For a groupoid $G$ with object space $X$, define elements $x, y \in X$ to be $G$ equivalent if there is an element of $G$ whose source is $x$ and whose range is $y$. This is readily seen to be an equivalence relation. A subset $A$ of $X$ is $G$-invariant if $A$ is saturated with respect to this equivalence relation, i.e., if any element $G$-equivalent to an element of $A$ is itself in $A$. We say a locally compact groupoid $G$ is minimal if there are no proper $G$-invariant open subsets.

Let $X$ be a compact Hausdorff space and $\sigma: X \rightarrow X$ a local homeomorphism. Then $C^{*}(R(X, \sigma))$ is simple iff $R(X, \sigma)$ is minimal ([58, Prop. II.4.6]). This can be reformulated as follows.

Proposition 4.1. Let $X$ be a compact metric space and $\sigma: X \rightarrow X$ a surjective local homeomorphism. Then $C^{*}(R(X, \sigma))$ is simple iff $\sigma$ is topologically exact. 
Proof. By compactness of $X, R(X, \sigma)$ is minimal iff $\sigma$ is topologically exact; cf. [39, Prop. 2.1].

We remark that surjectivity is not necessary in order for $C^{*}(R(X, \sigma))$ to be simple. If $\sigma$ is eventually surjective and is topologically exact on its eventual range, then a similar proof shows that $C^{*}(R(X, \sigma))$ is simple. Similar modifications are possible for the characterizations of simplicity for $C^{*}(X, \sigma)$ that follow.

Lemma 4.2. Let $X$ be a compact Hausdorff space and $\sigma: X \rightarrow X$ a surjective local homeomorphism. The following are equivalent:

(1) For every $x \in X$ and every open set $V$, there exist $n, m \in \mathbb{N}$ such that $\sigma^{n}(x) \in \sigma^{m}(V)$.

(2) $G(X, \sigma)$ is minimal.

(3) $\sigma$ is strongly transitive.

Proof. (11) $\Leftrightarrow$ (2) This is clear.

(11) $\Rightarrow$ (3) By (11), for every $x \in X$ and every open set $V$ of $X$, there exist $n, m \in \mathbb{N}$ such that $x \in \sigma^{-n} \sigma^{m}(V)$. This is equivalent to $\bigcup_{m, n} \sigma^{-n} \sigma^{m}(V)=X$. By compactness, this holds for a finite subcover, so $X=\bigcup_{i=1}^{k} \sigma^{-n_{i}} \sigma^{m_{i}}(V)$. Let $M=\max \left\{n_{1}, \ldots, n_{k}\right\}$. Then

$$
X=\sigma^{M}(X)=\bigcup_{i=1}^{k} \sigma^{M} \sigma^{-n_{i}} \sigma^{m_{i}}(V)=\bigcup_{i=1}^{k} \sigma^{M-n_{i}+m_{i}}(V) .
$$

Each $M-n_{i}+m_{i} \geq 0$, so $\sigma$ is strongly transitive.

(3) $\Rightarrow$ (11) Conversely, suppose $\sigma$ is strongly transitive. Let $V$ be an open subset of $X$. By strong transitivity, there exists $p$ such that $X=\bigcup_{k=0}^{p} \sigma^{k}(V)$, so (11) holds.

Let $X$ be a compact metric space. A local homeomorphism $\sigma: X \rightarrow X$ is essentially free if for every pair $m, n$ of distinct non-negative integers there is no open subset of $X$ on which $\sigma^{m}$ and $\sigma^{n}$ agree. The proof of the next result relies on Renault's result [59, Prop. 2.5] that if $\sigma: X \rightarrow X$ is an essentially free local homeomorphism and $G(X, \sigma)$ is minimal, then $C^{*}(X, \sigma)$ is simple.

Proposition 4.3. Let $X$ be a compact metric space and $\sigma: X \rightarrow X$ a surjective local homeomorphism. Then $C^{*}(X, \sigma)$ is simple iff $X$ has infinite cardinality and $\sigma$ is strongly transitive.

Proof. Assume first that $X$ is infinite and that $\sigma$ is strongly transitive. Then $G(X, \sigma)$ is minimal by Lemma 4.2. Furthermore, since $\sigma$ is surjective and transitive, there exists a point $p$ with a dense forward orbit; cf. [67, Thm. 5.9]. If $\sigma^{m}=\sigma^{n}$ on an open set $V$ with $m<n$, the orbit of $p$ must enter $V$, and thus is eventually periodic, and so in particular is finite. The finite orbit of $p$ cannot be dense in the infinite space $X$, so this is impossible. Thus $\sigma$ is essentially free, and $G(X, \sigma)$ is minimal, so $C^{*}(X, \sigma)$ is simple by [59, Prop. 2.5].

Conversely, suppose that $C^{*}(X, \sigma)$ is simple. Then there are no open $G(X, \sigma)$ invariant subsets by [58, Prop. II.4.5], so $G(X, \sigma)$ is minimal, and therefore $\sigma$ is strongly transitive by Lemma 4.2. Suppose that $X$ is finite. Surjectivity of $\sigma$ implies that $\sigma$ is bijective. Then as observed in the previous section, the map $(x, m) \mapsto\left(x, m, \sigma^{m} x\right)$ is a homeomorphism and isomorphism from the groupoid $X \times_{\sigma} \mathbb{Z}$ associated with the transformation group $\mathbb{Z}$ acting on $X$, onto $G(X, \sigma)$. 
Since $\mathbb{Z}$ does not act freely, the associated $\mathrm{C}^{*}$-algebra $C^{*}(G(X, \sigma)) \cong C(X) \times_{\sigma} \mathbb{Z}$ is not simple; cf. [11, p. 230]. This contradiction shows that $X$ is infinite.

For an alternative characterization for the case when $\sigma$ is a covering map, see [21, Thm. 11.2].

\section{C*-ALGEBRAS OF PIECEWISE MONOTONIC MAPS}

Definition 5.1. Let $\tau: I \rightarrow I$ be piecewise monotonic, and let $\sigma: X \rightarrow X$ be the associated local homeomorphism. We define $F_{\tau}=C^{*}(R(X, \sigma))$ and $O_{\tau}=C^{*}(X, \sigma)$.

We now give conditions for simplicity of $F_{\tau}$ and $O_{\tau}$.

Proposition 5.2. If $\tau: I \rightarrow I$ is piecewise monotonic and surjective, then $F_{\tau}$ is simple iff $\tau$ is topologically exact.

Proof. By Proposition 2.8, $\sigma$ is surjective, and $\tau$ is topologically exact iff $\sigma$ is topologically exact. Now the result follows from Proposition 4.1 .

Proposition 5.3. If $\tau: I \rightarrow I$ is piecewise monotonic and surjective, then $O_{\tau}$ is simple iff $\tau$ is transitive.

Proof. By Proposition 2.8, $\tau$ is surjective and is transitive iff $\sigma$ is strongly transitive. Now the result follows from Proposition 4.3 .

\section{Structural properties of $F_{\tau}$}

We are going to show that $F_{\tau}$ is always an AI-algebra (Definition 6.9 below), describe when $F_{\tau}$ is an AF-algebra, and give sufficient conditions for $F_{\tau}$ to be the direct sum of simple AF-algebras.

Notation. Let $X$ be a compact metric space, $\sigma: X \rightarrow X, n \in \mathbb{N}$, and assume there exists a partition of $X$ into clopen sets $X_{1}, \ldots, X_{p}$, and a clopen set $Y \subset X$, such that for each $i,\left.\sigma^{n}\right|_{X_{i}}$ is a homeomorphism from $X_{i}$ onto $Y$. For $1 \leq i \leq p$, let $\phi_{i}: Y \rightarrow X_{i}$ be the inverse of $\left.\sigma^{n}\right|_{X_{i}}$. For each pair of indices $i, j$ we define $\left(\phi_{i}, \phi_{j}\right)$ : $Y \rightarrow R_{n}(X, \sigma)$ by $\left(\phi_{i}, \phi_{j}\right)(y)=\left(\phi_{i}(y), \phi_{j}(y)\right)$. Let $\left\{e_{i j}\right\}$ be the standard matrix units of $M_{p}=M_{p}(\mathbb{C})$. If $Y$ is a compact metric space, we identify $C\left(Y, M_{n}\right)$ with $M_{n}(C(Y))$, so that each member $g$ of $C\left(Y, M_{n}\right)$ can be written as $g=\sum_{i j} g_{i j} e_{i j}$, where $g_{i j} \in C(Y)$.

Proposition 6.1. Assume $X$ is a compact metric space, $\sigma: X \rightarrow X$ a local homeomorphism, and $n \in \mathbb{N}$ such that $X$ admits a partition into clopen sets each mapped homeomorphically onto $Y \subset X$ by $\sigma^{n}$. With the notation above, the map $\pi: C^{*}\left(R_{n}(X, \sigma)\right) \rightarrow C\left(Y, M_{p}\right)$ given by

$$
\pi(f)=\sum_{i j}\left(f \circ\left(\phi_{i}, \phi_{j}\right)\right) e_{i j}
$$

is a ${ }^{*}$-isomorphism, so that $C^{*}\left(R_{n}\right) \cong C\left(Y, M_{p}\right)$.

Proof. $\pi$ is a ${ }^{*}$-isomorphism by a straightforward calculation.

Lemma 6.2. If $X$ is a compact metric space, and $\sigma: X \rightarrow X$ is a piecewise homeomorphism, then for each $n \in \mathbb{N}$, there exists a partition $B_{1}, \ldots, B_{q}$ of $X$ into clopen sets on which $\sigma^{n}$ is injective, and whose images are pairwise equal or disjoint. 
Proof. For each point $x \in X$ choose a clopen set on which $\sigma^{n}$ is injective, then a finite cover of $X$ by such sets, and then a partition of $X$ into clopen sets $A_{1}, \ldots, A_{p}$ on which $\sigma^{n}$ is injective. Now let $B_{1}, \ldots, B_{q}$ be the partition of $\sigma^{n} X$ into clopen sets generated by the sets $\sigma^{n}\left(A_{i}\right)$ for $1 \leq i \leq p$. Then the sets $\left\{\sigma^{-n}\left(B_{i}\right) \cap A_{j} \mid 1 \leq\right.$ $i \leq q, 1 \leq j \leq p\}$ form a partition $\mathcal{P}$ of $X$ such that the images of the partition members under $\sigma^{n}$ are disjoint or equal.

Corollary 6.3. Let $X$ be a compact metric space and $\sigma: X \rightarrow X$ a piecewise homeomorphism, $n \in \mathbb{N}$. Choose a partition of $X$ into clopen subsets on which $\sigma^{n}$ is injective, with the images of these sets being either equal or disjoint. Let $Y_{1}, \ldots, Y_{q}$ be the collection of the distinct images, and let $n_{i}$ be the number of inverse images of each point in $Y_{i}$. Then $C^{*}\left(R_{n}\right) \cong \bigoplus_{i} C\left(Y_{i}, M_{n_{i}}\right)$.

Proof. The sets $\left\{V_{i}=\sigma^{-n} Y_{i} \mid 1 \leq i \leq q\right\}$ form a partition of $X$ into clopen $R_{n}(X, \sigma)$-invariant subsets. Let $\left.R_{n}\right|_{V_{i}}$ denote $R_{n} \cap\left(V_{i} \times V_{i}\right)$. Then $C^{*}\left(R_{n}\right) \cong$ $\bigoplus_{i} C^{*}\left(\left.R_{n}\right|_{V_{i}}\right)$. Now the corollary follows by repeated application of Proposition 6.1, with $V_{i}$ in place of $X$ and $Y_{i}$ in place of $Y$.

By "interval" we will always mean a non-degenerate interval, i.e., not a single point.

Lemma 6.4. If $X$ is a compact subset of $\mathbb{R}$, then there is a decreasing sequence $\left\{X_{n}\right\}$ of subsets of $\mathbb{R}$ such that $\bigcap_{n} X_{n}=X$, and such that each $X_{n}$ is a finite union of closed intervals. For such a sequence, $C(X)$ is the inductive limit of the algebras $C\left(X_{n}\right)$, with the connecting maps $C\left(X_{n}\right) \rightarrow C\left(X_{n+1}\right)$ given by the restriction map.

Proof. Let $[a, b]$ be a closed interval containing $X$. Write $\mathbb{R} \backslash X$ as a countable disjoint union of open intervals $V_{1}, V_{2}, \ldots$ Let $X_{n}=[a, b] \backslash \bigcup_{i=1}^{n} \phi_{n}\left(V_{i}\right)$, where for each $n$ and each open interval $J, \phi_{n}(J)$ is an open interval with the same midpoint as $J$, but with length shrunk by a factor $(1-1 / n)$. Then each $X_{n}$ is a finite union of closed intervals. (The shrinking given by $\phi_{n}$ is needed to insure that these intervals are non-degenerate.) The sets $X_{n}$ are a decreasing sequence with intersection equal to $X$. Thus $X$ is the projective limit $X_{1} \leftarrow X_{2} \cdots$, where the connecting maps are the inclusion maps. Therefore $C(X)$ is the inductive limit of the sequence $C\left(X_{1}\right) \rightarrow C\left(X_{2}\right) \rightarrow \cdots$.

If $\sigma: X \rightarrow X$ is the local homeomorphism associated with a piecewise monotonic map $\tau$, then $X$ need not be totally disconnected. We recall from 63] the characterization of total disconnectedness for $X$.

Definition 6.5. An interval $J$ is a homterval for a piecewise monotonic map $\tau$ : $I \rightarrow I$ if $\tau^{n}$ is a homeomorphism on $J$ for all $n$.

Example 6.6. A polynomial of degree $\geq 2$ has homtervals iff it has an attractive periodic orbit; cf. [63, Prop. 5.7].

Proposition 6.7. Let $\tau: I \rightarrow I$ be piecewise monotonic, $\sigma: X \rightarrow X$ the associated local homeomorphism, and $C$ the associated partition for $\tau$. Then the following are equivalent:

(1) $X$ is totally disconnected.

(2) The generalized orbit of $C$ is dense in $[0,1]$.

(3) $\tau$ has no homtervals.

In particular, these hold if $\tau$ is transitive. 
Proof. [63, Prop. 5.8].

Recall that an AF-algebra is a $\mathrm{C}^{*}$-algebra which is the inductive limit of a sequence of finite-dimensional $\mathrm{C}^{*}$-algebras. The following can also be derived from [58, Prop. III.1.15].

Proposition 6.8. If a compact Hausdorff space $X$ is totally disconnected and $\sigma$ : $X \rightarrow X$ is a local homeomorphism, then $C^{*}(R(X, \sigma))$ is an AF-algebra.

Proof. It suffices to show that $C^{*}\left(R_{n}\right)$ is AF for each $n$. Since $X$ is totally disconnected, then $\sigma$ is a piecewise homeomorphism. By Corollary 6.3, $C^{*}\left(R_{n}\right)$ is isomorphic to $\bigoplus_{i} C\left(Y_{i}, M_{n}\right)$, where each $Y_{i}$ is a clopen subset of $X$. Since each $Y_{i}$ is totally disconnected, then each $C\left(Y_{i}, M_{n}\right)$ is an AF-algebra, and thus $C^{*}\left(R_{n}\right)$ and $C^{*}(R(X, \sigma))$ are $\mathrm{AF}$-algebras.

The converse of Proposition 6.8 is false, as was shown by Blackadar 4, Thm. 7.2.1].

Definition 6.9. A $\mathrm{C}^{*}$-algebra is an interval algebra if it is of the form $C([0,1], A)$ where $A$ is a finite-dimensional $\mathrm{C}^{*}$-algebra, and is an $A I$-algebra if it is an inductive limit of interval algebras.

Proposition 6.10. If $\tau: I \rightarrow I$ is piecewise monotonic, then $F_{\tau}$ is an AI-algebra. It is an AF-algebra iff $\tau$ has no homterval. In particular, this is true if $\tau$ is transitive.

Proof. First, we show that $F_{\tau}$ is an AI-algebra. Each subalgebra $C^{*}\left(R_{n}\right)$ is isomorphic to a sum of algebras of the form $C\left(Y, M_{k}\right)$ for $Y$ a compact subset of $\mathbb{R}$ (Corollary 6.3). Here $C(Y)$ is the inductive limit of algebras $C\left(Y_{i}\right)$ where $Y_{i}$ is a finite union of closed intervals (Lemma 6.4), so $C\left(Y, M_{k}\right)$ is the inductive limit of $C\left(Y_{i}, M_{k}\right)$, and thus is an AI-algebra. Hence $\left\{C^{*}\left(R_{n}\right)\right\}$ is an increasing sequence of AI-algebras and is dense in $C^{*}(R(X, \sigma))$, so the latter is an AI-algebra. (An inductive limit of AI-algebras is an AI-algebra, since AI-algebras admit a local characterization; cf. Elliott's proof of the analogous result for AT-algebras [19, Lemma 4.2 and Thm. 4.3].)

If $\tau$ has no homtervals, then $X$ is totally disconnected (Proposition 6.7), and so $F_{\tau}=C^{*}(R(X, \sigma))$ is $\mathrm{AF}$ (Proposition 6.8).

Now suppose that $\tau$ has a homterval $J$. Let $\pi: X \rightarrow I$ be the collapse map. Then $\pi^{-1}(J) \subset X$ is connected, and $\sigma^{n}$ is injective on it for all $n$. The same is true for the connected component $J^{\prime}$ of $X$ containing $\pi^{-1}(J)$, and $J^{\prime}$ is homeomorphic to $[0,1]$.

Thus the equivalence relation $R(X, \sigma)$ restricted to $J^{\prime}$ is trivial. Let $p$ be the projection corresponding to $J^{\prime}$ (where $J^{\prime}$ is viewed as a subset of the diagonal of $R(X, \sigma))$. Since no distinct points in $J^{\prime}$ are equivalent, $p C_{c}(R(X, \sigma)) p$ is the set of functions in $C_{c}(R(X, \sigma))$ with support in the diagonal intersected with $J^{\prime} \times J^{\prime}$. This is then isomorphic to $C\left(J^{\prime}\right)$. Since this is dense in $p C^{*}(R(X, \sigma)) p$ and injections are isometric, then $p C^{*}(R(X, \sigma)) p \cong C\left(J^{\prime}\right)$. If $C^{*}(R(X, \sigma))$ were an AF-algebra, then the corner $p C^{*}(R(X, \sigma)) p$ would also be AF. Since $C\left(J^{\prime}\right)$ is not an AF-algebra, then neither is $C^{*}(R(X, \sigma))$.

It is also possible to give a constructive proof of the fact that $F_{\tau}$ is an AI-algebra, by showing that $F_{\tau}$ is isomorphic to the algebra $A_{\tau}$ defined in [63, 112 ]; cf. [14, Thm. 8.1]. 
Definition 6.11. A piecewise monotonic map $\tau: I \rightarrow I$ is essentially injective if there is no pair $U, V$ of disjoint open sets such that $\tau(U)=\tau(V)$.

The map $\tau$ will be essentially injective iff the associated local homeomorphism $\sigma: X \rightarrow X$ is injective; cf. [63, Lemma 11.3].

Theorem 6.12. If $\tau: I \rightarrow I$ is transitive and is not essentially injective, and $\sigma: X \rightarrow X$ is the associated local homeomorphism, then there is a positive integer $n$ and a partition of $X$ into clopen sets $X_{1}, \ldots, X_{n}$ cyclically permuted by $\sigma$, such that $\sigma^{n}$ restricted to each set $X_{i}$ is topologically exact.

Proof. [64, Thm. 4.5].

Corollary 6.13. Let $\tau: I \rightarrow I$ be piecewise monotonic, transitive, and not essentially injective, and let $X_{1}, \ldots, X_{n}$ be a partition of $X$ as in Theorem 6.12. Then $F_{\tau}=A_{1} \oplus A_{2} \oplus \cdots \oplus A_{n}$, where for each $i, A_{i} \cong C^{*}\left(R\left(X_{i}, \sigma^{n}\right)\right)$ is a simple $A F$-algebra. For each index $i$, the canonical endomorphism $\Phi$ of $F_{\tau}$ maps $A_{i}$ into $A_{i-1 \bmod n}$.

Proof. Since $R_{k} \subset R_{k+1}$ for all $k$, then $R(X, \sigma)=\bigcup_{k} R_{k n}=R\left(X, \sigma^{n}\right)$. Since $\sigma$ cyclically permutes $X_{1}, \ldots, X_{n}$, then for each $i,\left\{(x, y) \in R(X, \sigma) \cap\left(X_{i} \times X_{i}\right)\right.$ | $\left.\sigma^{n}(x)=\sigma^{n}(y)\right\}$ is a clopen invariant subset of $R(X, \sigma)$, and is isomorphic as a locally compact groupoid to $R\left(X_{i}, \sigma^{n}\right)$. For $1 \leq i \leq n$, let $A_{i}$ be the closure in $F_{\tau}$ of the algebra of functions with compact support in $R(X, \sigma) \cap\left(X_{i} \times X_{i}\right)$. Then $F_{\tau}=A_{1} \oplus A_{2} \oplus \cdots \oplus A_{n}$. Since $\sigma$ permutes $X_{1}, \ldots, X_{n}$ cyclically, then $(x, y) \in X_{i} \times X_{i}$ is in $R(X, \sigma)$ iff $\sigma^{j} x=\sigma^{j} y$ for some $j$ a multiple of $n$. Thus $A_{i} \cong C^{*}\left(R\left(X_{i}, \sigma^{n}\right)\right)$ for $1 \leq i \leq n$. Since $\sigma^{n}$ is topologically exact on $X_{i}$, then each $A_{i}$ is a simple AF-algebra by Proposition 4.1 and Proposition 6.10. Since $\sigma$ maps $X_{i}$ to $X_{i+1 \bmod n}$, by the definition of the canonical endomorphism $\Phi$ of $F_{\tau}$, when $f \in C_{c}(R(X, \sigma))$ has support in $X_{i}$, then $\Phi(f)$ will have support in $X_{i-1 \bmod n}$. Thus $\Phi$ will map $A_{i}$ into $A_{i-1} \bmod n$.

\section{A DYNAMiCAL DESCRIPTION OF $K_{0}\left(F_{\tau}\right)$}

Our goal in this section is to show that $K_{0}\left(F_{\tau}\right)$ is isomorphic to the dynamically defined dimension group $D G(\tau)$ defined in 63. See [23] or [16] for background on dimension groups.

An ordered abelian group $G$ is unperforated if for each positive integer $n$ and $g \in G$, if $n g \in G^{+}$, then $g \in G^{+}$. An ordered abelian group $G$ satisfies the Riesz decomposition property if whenever $g_{1}, g_{2}, h_{1}, h_{2} \in G$ with $g_{i} \leq h_{j}$ for $i, n=1,2$, then there exists $f$ with $g_{i} \leq f \leq h_{j}$ for $i, j=1,2$.

Definition 7.1. An ordered abelian group $G$ is a dimension group if $G$ is unperforated and has the Riesz decomposition property.

If $G$ is countable, then by a result of Effros-Handelman-Shen [17], $G$ is a dimension group iff $G$ is an inductive limit of a sequence of ordered groups $\mathbb{Z}^{n_{k}}$; cf. [17. If $X$ is any compact Hausdorff space, then $C(X, \mathbb{Z})$ (with the pointwise ordering) is easily seen to be a dimension group.

Definition 7.2. Let $X$ be a compact Hausdorff space and $\sigma: X \rightarrow X$ a local homeomorphism. Then the transfer map $\mathcal{L}_{\sigma}: C(X, \mathbb{Z}) \rightarrow C(X, \mathbb{Z})$ is defined by

$$
\mathcal{L}_{\sigma}(f)(x)=\sum_{\sigma y=x} f(y)
$$


(We will write $\mathcal{L}$ in place of $\mathcal{L}_{\sigma}$ when the meaning is clear from the context.)

By compactness of $X$ and the definition of a local homeomorphism, each fiber $\sigma^{-1}(x)$ is finite, so the sum in the definition of $\mathcal{L}$ is finite. Note that $\mathcal{L}$ is a positive map, and if $\sigma$ is injective on a clopen set $E$, then

$$
\mathcal{L}_{\sigma}\left(\chi_{E}\right)=\chi_{\sigma(E)} .
$$

The transfer map will play a central role in our dynamical description of $K_{0}\left(F_{\tau}\right)$.

Definition 7.3. Let $X$ be a compact metric space, and $\sigma: X \rightarrow X$ a piecewise homeomorphism. Define an equivalence relation on $C(X, \mathbb{Z})$ by $f \sim g$ if $\mathcal{L}_{\sigma}^{n} f=\mathcal{L}_{\sigma}^{n} g$ for some $n \geq 0$, and denote equivalence class by square brackets. Define $[f]+[g]=$ $[f+g]$, and $[f] \geq 0$ if $\mathcal{L}_{\sigma}^{n} f \geq 0$ for some $n \geq 0$. The set of equivalence classes is an ordered abelian group, denoted $G_{\sigma}$. If $\tau: I \rightarrow I$ is piecewise monotonic and $\sigma: X \rightarrow X$ is the associated local homeomorphism, then $D G(\tau)$ is defined to be $G_{\sigma}$. (We will see below that $G_{\sigma}$ and $D G(\tau)$ are dimension groups.)

Lemma 7.4. Let $X$ be a compact Hausdorff space, $\sigma: X \rightarrow X$ a piecewise homeomorphism, and $Y=\sigma(X)$. Then $\mathcal{L}(C(X, \mathbb{Z}))=\{f \in C(X, \mathbb{Z}) \mid \operatorname{supp} f \subset$ $Y\}, \mathcal{L}(C(X, \mathbb{Z}))^{+}=\mathcal{L}\left(C(X, \mathbb{Z})^{+}\right)$, and $\mathcal{L}(C(X, \mathbb{Z})) \cong C(Y, \mathbb{Z})$. In particular, $\mathcal{L}(C(X, \mathbb{Z}))$ is a dimension group.

Proof. [63, Lemmas 3.5, 3.9].

Proposition 7.5. Let $X$ be a compact subset of $\mathbb{R}$, and let $\sigma: X \rightarrow X$ be a piecewise homeomorphism. Then $G_{\sigma}$ is isomorphic to the inductive limit

$$
C(X, \mathbb{Z}) \stackrel{\mathcal{L}}{\longrightarrow} \mathcal{L}(C(X, \mathbb{Z})) \stackrel{\mathcal{L}}{\longrightarrow} \mathcal{L}^{2}(C(X, \mathbb{Z})) \stackrel{\mathcal{L}}{\longrightarrow} \cdots
$$

and is a dimension group if equipped with the positive cone $G_{\sigma}^{+}=\{[f] \mid f \in$ $\left.C(X, \mathbb{Z})^{+}\right\}$. The map $\mathcal{L}_{*}: G_{\sigma} \rightarrow G_{\sigma}$ is positive and injective, and is an order automorphism iff $\sigma$ is eventually surjective.

Proof. [63, Lemma 3.8, Cor. 3.12, and Prop. 4.5].

We are going to show in Proposition 7.7 that $K_{0}(C(X))=C(X, \mathbb{Z})$ for $X$ a compact subset of $\mathbb{R}$. We will do this by computing appropriate inductive limits.

Lemma 7.6. If $X_{1}, X_{2}, \ldots$ is a decreasing sequence of compact subsets of a Hausdorff topological space, and $X=\bigcap_{n} X_{n}$, then the dimension group $C(X, \mathbb{Z})$ is the inductive limit of the sequence $C\left(X_{1}, \mathbb{Z}\right) \rightarrow C\left(X_{2}, \mathbb{Z}\right) \rightarrow \cdots \rightarrow C\left(X_{n}, \mathbb{Z}\right) \rightarrow \cdots$, where the connecting maps are the restriction maps.

Proof. Let $G$ be the inductive limit of the sequence $C\left(X_{n}, \mathbb{Z}\right)$ of dimension groups, thought of as sequences $f_{1}, f_{2}, \ldots$ where $f_{i} \in C\left(X_{i}, \mathbb{Z}\right)$, and such that $f_{i+1}=\left.f_{i}\right|_{X_{i+1}}$ for all but finitely many $i$. Two such sequences are identified if they agree for all but finitely many terms. If $f_{i+1}=\left.f_{i}\right|_{X_{i+1}}$ for all $i \geq n$, we represent the equivalence class of this sequence by $\left[f_{n}, n\right]$, which will be the same as $[g, m]$ if $\left.f_{n}\right|_{X_{k}}=\left.g\right|_{X_{k}}$ for some $k>\max \{m, n\}$.

Suppose $f \in C\left(X_{n}\right)$ and $\left.f\right|_{X}=0$. Fix $\epsilon>0$. We claim

$$
\text { there exists } k \geq n \text { such that }|f|<\epsilon \text { on } X_{k} \text {. }
$$

Indeed, let $G_{k}=\left\{x \in X_{k}|| f(x) \mid \geq \epsilon\right\}$; then $G_{1}, G_{2}, \ldots$ is a nested decreasing sequence of compact sets with empty intersection, so some $G_{k}$ is empty, which proves $(*)$. 
Define $\Psi: G \rightarrow C(X, \mathbb{Z})$ by $\Psi([f, n])=\left.f\right|_{X}$. Suppose that $\Psi([f, n])=0$, i.e., $f \in C\left(X_{n}, \mathbb{Z}\right)$ and $\left.f\right|_{X}=0$. Choosing $\epsilon<1$ in (1), since $f$ has integer values, we conclude that there exists $k>n$ such that $\left.f\right|_{X_{k}}=0$, so $[f, n]=0$. Thus $\Psi$ is injective.

To see that $\Psi$ is surjective, let $p$ be the characteristic function of a clopen set $E \subset X$. Choose $f \in C\left(X_{1}\right)$ such that $0 \leq f \leq 1$ and such that $\left.f\right|_{X}=p$. Then $f-f^{2}=0$ on $X$, so by (1), there exists $k \geq 1$ such that $\left|f-f^{2}\right| \leq 3 / 16$ on $X_{k}$. Then $f\left(X_{k}\right)$ is contained in $[0,1 / 4] \cup[3 / 4,1]$. Choose a continuous function $\phi:[0,1] \rightarrow$ $[0,1]$ such that $\phi=0$ on $[0,1 / 4]$, and $\phi=1$ on $[3 / 4,1]$. Then $h=\left.(\phi \circ f)\right|_{X_{k}}$ is a projection in $C\left(X_{k}\right)$, and $\left.h\right|_{X}=\phi \circ p=p$. Thus $\Psi([h, k])=p$, so $\Psi$ is surjective, which completes the proof that $C(X, \mathbb{Z})$ is the desired inductive limit.

Proposition 7.7. If $X$ is a compact subset of $\mathbb{R}$, then there is a unique isomorphism $K_{0}(C(X)) \cong C(X, \mathbb{Z})$, which for each clopen set $E \subset X$ takes the class $\left[\chi_{E}\right] \in K_{0}(C(X))$ to $\chi_{E} \in C(X, \mathbb{Z})$.

Proof. Choose $X_{1} \supset X_{2} \cdots$ so that each $X_{i}$ is a finite union of closed intervals, and such that $\bigcap_{n} X_{n}=X$; cf. Lemma 6.4. For a closed interval $J$, we have $K_{0}(C(J))=\mathbb{Z}$, with a generator being the function constantly 1 on $J$, so we can identify $K_{0}(C(J))$ with $C(J, \mathbb{Z})$. Therefore $K_{0}\left(C\left(X_{n}\right)\right) \cong C\left(X_{n}, \mathbb{Z}\right)$, and this isomorphism takes the class $\left[\chi_{E}\right] \in K_{0}\left(C\left(X_{n}\right)\right)$ to $\chi_{E} \in C\left(X_{n}, \mathbb{Z}\right)$. Now the result follows from the fact that $C(X)$ is the inductive limit of the sequence $C\left(X_{1}\right) \rightarrow$ $C\left(X_{2}\right) \rightarrow \ldots$, continuity of $K_{0}$ with respect to inductive limits, and Lemma 7.6.

The second author would like to thank Jack Spielberg for helpful conversations regarding the proof of Proposition 7.7 .

For each clopen subset $E$ of $X$, we write $\widetilde{E}$ for the corresponding subset $\{(x, x) \in$ $X \times X \mid x \in E\}$ of the diagonal of $R(X, \sigma)$, and $\left[\chi_{\widetilde{E}}\right]$ denotes the class of the projection $\chi_{\widetilde{E}}$ in $K_{0}\left(C^{*}\left(R_{n}\right)\right)$.

Lemma 7.8. Let $X$ be a compact subset of $\mathbb{R}$, and let $\sigma: X \rightarrow X$ be a piecewise homeomorphism. For each $k \in \mathbb{N}$, there is a unique isomorphism of $K_{0}\left(C^{*}\left(R_{k}\right)\right)$ onto $\mathcal{L}^{k} C(X, \mathbb{Z})$ taking $\left[\chi_{\widetilde{E}}\right]$ to $\mathcal{L}^{k} \chi_{E}$ for each clopen set $E \subset X$, and the elements $\left[\chi_{\widetilde{E}}\right]$ generate $K_{0}\left(C^{*}\left(R_{k}\right)\right)$.

Proof. We may assume without loss of generality that $k=1$. (Otherwise just replace $\sigma$ by $\sigma^{k}$, and observe that $\mathcal{L}_{\sigma}^{k}=\mathcal{L}_{\sigma^{k}}$ and $R_{k}(X, \sigma)=R_{1}\left(X, \sigma^{k}\right)$.) By Lemma 6.2, we can construct a partition of $X$ into clopen sets on each of which $\sigma$ is injective, and whose images are either equal or disjoint. Let $Y_{1}, \ldots, Y_{p}$ be the distinct images of these sets, and let $W_{i}=\sigma^{-1}\left(Y_{i}\right)$ for $1 \leq i \leq p$. By construction, for each $i, W_{i}$ admits a finite partition into clopen sets mapped homeomorphically by $\sigma$ onto $Y_{i}$. It suffices to prove the statement of the lemma with $W_{i}$ in place of $X$ for $1 \leq i \leq p$. Thus without loss of generality, we may assume $p=1$.

Let $X_{1}, \ldots, X_{n}$ be a partition of $X$ into clopen subsets on which $\sigma$ is 1-1, such that $\sigma\left(X_{i}\right)=Y \subset X$ for $1 \leq i \leq n$. We have isomorphisms

$$
K_{0}\left(C^{*}\left(R_{1}\right)\right) \cong K_{0}\left(C\left(Y, M_{n}\right)\right) \cong K_{0}(C(Y)) \cong C(Y, \mathbb{Z}) \cong \mathcal{L} C(X, \mathbb{Z}) .
$$

Here the first isomorphism is induced by the isomorphism of $C^{*}\left(R_{1}\right)$ and $C\left(Y, M_{n}\right)$ given in Proposition 6.1. The second is induced by the inverse of the isomorphism from $C(Y)$ onto $C(Y) e_{11}$ given by $f \mapsto f e_{11}$. The third isomorphism is given by 
Proposition 7.7. Finally, by Lemma 7.4, extending functions in $C(Y, \mathbb{Z})$ to functions in $X$ that are zero off $Y$ gives an isomorphism $C(Y, \mathbb{Z}) \cong \mathcal{L} C(X, \mathbb{Z})$.

Now suppose $E$ is a clopen subset of the partition member $X_{1}$. Then under the sequence of isomorphisms in (2),

$\left[\chi_{\widetilde{E}}\right] \mapsto\left[\chi_{\widetilde{E}} \circ\left(\phi_{1}, \phi_{1}\right) e_{11}\right]=\left[\chi_{\sigma(E)} e_{11}\right] \mapsto\left(\left[\chi_{\sigma(E)}\right] \in C(Y, \mathbb{Z})\right) \mapsto \mathcal{L} \chi_{E} \in \mathcal{L} C(X, \mathbb{Z})$.

Since the functions $\mathcal{L} \chi_{E}$ generate the group $\mathcal{L} C(X, \mathbb{Z})$, it follows that the classes $\left[\chi_{\tilde{E}}\right]$ generate $K_{0}\left(C^{*}\left(R_{1}\right)\right)$, which proves the uniqueness statement in the lemma.

Theorem 7.9. Let $X$ be a compact subset of $\mathbb{R}$, and let $\sigma: X \rightarrow X$ be a piecewise homeomorphism. Then there is a unique isomorphism $K_{0}\left(C^{*}(R(X, \sigma))\right) \rightarrow$ $G_{\sigma}$ taking $\left[\widetilde{\chi}_{E}\right]$ to $\left[\chi_{E}\right]$ (for each clopen subset $E$ of $X$ ). If $\sigma$ is surjective and $\Phi$ is the canonical endomorphism of $C^{*}(R(X, \sigma))$, then the automorphism $\Phi_{*}$ of $K_{0}\left(C^{*}(R(X, \sigma))\right)$ is carried to the automorphism $\left(\mathcal{L}_{\sigma}\right)_{*}^{-1}$ on $G_{\sigma}$.

Proof. If we identify $X$ with the diagonal of $R(X, \sigma)$, i.e., with $R_{0}(X, \sigma)$, for each $n \in \mathbb{N}$ we have the commutative diagram

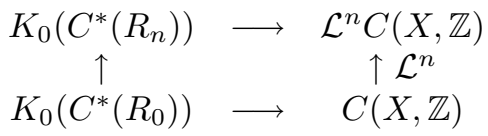

where the vertical arrow on the left is induced by the inclusion of $C^{*}\left(R_{0}\right)$ in $C^{*}\left(R_{n}\right)$, and the horizontal arrows are isomorphisms given in Lemma 7.8. Since $\mathcal{L}^{n}: C(X, \mathbb{Z}) \rightarrow \mathcal{L}^{n} C(X, \mathbb{Z})$ is surjective, then the map $K_{0}\left(C^{*}\left(R_{0}\right)\right) \rightarrow K_{0}\left(C^{*}\left(R_{n}\right)\right)$ is also surjective. It follows that the following diagram commutes:

$$
\begin{array}{ccc}
K_{0}\left(C^{*}\left(R_{n+1}\right)\right) & \longrightarrow & \mathcal{L}^{n+1} C(X, \mathbb{Z}) \\
\uparrow & & \uparrow \mathcal{L} \\
K_{0}\left(C^{*}\left(R_{n}\right)\right) & \longrightarrow & \mathcal{L}^{n} C(X, \mathbb{Z})
\end{array}
$$

Since $C^{*}(R(X, \sigma))$ is the inductive limit of the sequence $\left\{C^{*}\left(R_{n}(X, \sigma)\right)\right\}$, then $K_{0}\left(C^{*}(R(X, \sigma))\right)$ is the inductive limit of the sequence $\left\{K_{0}\left(C^{*}\left(R_{n}\right)\right)\right\}$. By virtue of the commutative diagram above, this is the same as the inductive limit of the sequence $\mathcal{L}: \mathcal{L}^{n} C(X, \mathbb{Z}) \rightarrow \mathcal{L}^{n+1} C(X, \mathbb{Z})$, which is in turn isomorphic to $G_{\sigma}$; cf. Proposition 7.5 .

Finally, we show that $\mathcal{L}_{*} \Phi_{*}$ is the identity map on $K_{0}\left(C^{*}(R(X, \sigma))\right)$. For this purpose, it suffices to prove that $\mathcal{L}_{*} \Phi_{*}\left[\chi_{\tilde{Y}}\right]=\left[\chi_{\tilde{Y}}\right]$ for every clopen $Y \subset X$. Without loss of generality, $Y$ is contained in one of the sets $Y_{i}$ in Corollary 6.3 (applied with $n=1$ ), so that there are clopen sets $E_{1}, \ldots, E_{p}$ each mapped bijectively by $\sigma$ onto $Y$. Then $\Phi\left(\chi_{\tilde{Y}}\right)=(1 / p) \sum \chi_{E_{i j}}$, where $E_{i j}=\left\{(x, y) \in E_{i} \times E_{j} \mid \sigma x=\sigma y\right\}$. Letting $w=(1 / \sqrt{p}) \sum_{i} \chi_{E_{1 i}}$, then $w w^{*}=\chi_{E_{11}}$ and

$$
w^{*} w=(1 / p) \sum \chi_{E_{i j}}=\Phi\left(\chi_{\tilde{Y}}\right) .
$$

Thus

$$
\mathcal{L}_{*} \Phi_{*}\left[\chi_{\tilde{Y}}\right]=\mathcal{L}_{*}\left[\chi_{E_{11}}\right]=\left[\chi_{\tilde{Y}}\right],
$$

which completes the proof of the theorem.

Corollary 7.10. If $\tau: I \rightarrow I$ is piecewise monotonic, then $K_{0}\left(F_{\tau}\right) \cong D G(\tau)$. If $\tau$ is surjective, and $\Phi$ is the canonical endomorphism of $F_{\tau}$, then the action of $\Phi_{*}$ on $K_{0}\left(F_{\tau}\right)$ corresponds to $\mathcal{L}_{*}^{-1}$ on $D G(\tau)$. 
Proof. This follows from Theorem 7.9 and the definitions $F_{\tau}=C^{*}(R(X, \sigma))$ and $D G(\tau)=G_{\sigma}$.

A continuous map $\tau: I \rightarrow I$ is unimodal if for some $c \in I, \tau$ increases on $[0, c]$ and decreases on $[c, 1]$. (If instead $\tau$ decreases and then increases, the map $\phi(x)=1-x$ is a conjugacy from $\tau$ onto a unimodal map.)

Corollary 7.11. If $\tau_{i}: I \rightarrow I$ are unimodal and transitive for $i=1,2$, and $\left(K_{0}\left(F_{\tau_{1}}\right),\left(\Phi_{1}\right)_{*}\right) \cong\left(K_{0}\left(F_{\tau_{2}}\right),\left(\Phi_{2}\right)_{*}\right)$, then $\tau_{1}$ and $\tau_{2}$ are conjugate.

Proof. If $\left(K_{0}\left(F_{\tau_{1}}\right),\left(\Phi_{1}\right)_{*}\right) \cong\left(K_{0}\left(F_{\tau_{2}}\right),\left(\Phi_{2}\right)_{*}\right)$, then by Corollary 17.10, the dimension triples $\left(D G\left(\tau_{1}\right), D G\left(\tau_{1}\right)^{+}, \mathcal{L}_{*}\right)$ and $\left(D G\left(\tau_{2}\right), D G\left(\tau_{2}\right)^{+}, \mathcal{L}_{*}\right)$ are isomorphic, so by [64, Cor. 8.3], the maps $\tau_{1}$ and $\tau_{2}$ are conjugate.

When $\tau$ is transitive, the dimension group $D G(\tau)$ has a unique state scaled by the canonical automorphism $\mathcal{L}_{*}=\Phi_{*}^{-1}$. The scaling factor is $\exp \left(h_{\tau}\right)$, where $h_{\tau}$ is the topological entropy of $\tau$; cf. 64, Thm. 5.3]. Thus one can recover $h_{\tau}$ from the dimension group together with the canonical automorphism.

Corollary 7.12. If $\tau_{i}: I \rightarrow I$ are piecewise monotonic and transitive for $i=1,2$, and $\left(K_{0}\left(F_{\tau_{1}}\right),\left(\Phi_{1}\right)_{*}\right) \cong\left(K_{0}\left(F_{\tau_{2}}\right),\left(\Phi_{2}\right)_{*}\right)$, then $h_{\tau_{1}}=h_{\tau_{2}}$.

Proof. If $\left(K_{0}\left(F_{\tau_{1}}\right),\left(\Phi_{1}\right)_{*}\right) \cong\left(K_{0}\left(F_{\tau_{2}}\right),\left(\Phi_{2}\right)_{*}\right)$, then by Corollary 17.10, the dimension triples $\left(D G\left(\tau_{1}\right), D G\left(\tau_{1}\right)^{+}, \mathcal{L}_{*}\right)$ and $\left(D G\left(\tau_{2}\right), D G\left(\tau_{2}\right)^{+}, \mathcal{L}_{*}\right)$ are isomorphic, so by [64, Cor. 5.4], the maps $\tau_{1}$ and $\tau_{2}$ have the same topological entropy.

\section{The Module StruCture of $K_{0}\left(F_{\tau}\right)$}

If $\tau:[0,1] \rightarrow[0,1]$ is piecewise monotonic and surjective, then the canonical endomorphism $\Phi$ of $F_{\tau}$ induces an automorphism $\Phi_{*}$ of $K_{0}\left(F_{\tau}\right)$; cf. Corollary 7.10, Under the isomorphism of $K_{0}\left(F_{\tau}\right)$ with $D G(\tau), \Phi_{*}^{-1}$ is carried to $\mathcal{L}_{*}$.

The automorphism $\mathcal{L}_{*}$ gives $K_{0}\left(F_{\tau}\right) \cong D G(\tau)$ the structure of a $\mathbb{Z}\left[t, t^{-1}\right]$ module. In 63], a finite set of generators for this module is described. For later use, we summarize those results.

As usual, $\sigma: X \rightarrow X$ denotes the local homeomorphism associated with $\tau$. If $D$ is a finite subset of $\mathbb{R}$, we will say distinct points $x, y \in D$ are adjacent in $D$ if there is no element of $D$ between them. Recall that $I(c, d)=\left[c^{+}, d^{-}\right]_{X}$ for $c, d \in I_{1}$, where $I_{1}$ is the generalized orbit in $I$ of the partition points $a_{0}, \ldots, a_{n}$ for $\tau$. We will identify clopen subsets of $X$ with their characteristic functions, so $I(c, d)$ will be viewed as an element of $D G(\tau)$. For the definition of $\tau_{1}, \ldots, \tau_{n}$ in Theorem 8.1, see Definition 2.1.

Theorem 8.1. Let $\tau: I \rightarrow I$ be piecewise monotonic and surjective, with associated partition $a_{0}, a_{1}, \ldots, a_{n}$. Let $M$ be any member of the set $\left\{\tau_{1}\left(a_{0}\right), \tau_{1}\left(a_{1}\right), \ldots\right.$, $\left.\tau_{n}\left(a_{n-1}\right), \tau_{n}\left(a_{n}\right)\right\}$. Let

$$
\mathcal{J}_{1}=\left\{I(c, d) \mid c, d \text { are adjacent points in }\left\{a_{0}, a_{1}, \ldots, a_{n}, M\right\}\right\},
$$

and let $\mathcal{J}_{2}$ be the set of intervals corresponding to jumps at partition points, i.e.,

$$
\mathcal{J}_{2}=\left\{I\left(\tau_{i}\left(a_{i}\right), \tau_{i+1}\left(a_{i}\right)\right) \mid 1 \leq i \leq n-1\right\} .
$$

Then $D G(\tau)$ is generated as a module by $\mathcal{J}_{1} \cup \mathcal{J}_{2}$.

Proof. 63, Thm. 6.2]. 
Corollary 8.2. If $\tau: I \rightarrow I$ is a continuous, surjective piecewise monotonic map, with associated partition $a_{0}, a_{1}, \ldots, a_{n}$, then $D G(\tau)$ is generated as a module by $I\left(a_{0}, a_{1}\right), I\left(a_{1}, a_{2}\right), \ldots, I\left(a_{n-1}, a_{n}\right)$.

Proof. [63, Cor. 6.3].

\section{K-GROuPS OF $O_{\tau}$}

Let $A$ be a unital $\mathrm{C}^{*}$-algebra, and $\alpha: A \rightarrow A$ a proper corner endomorphism, i.e., $q=\alpha(1)$ is a proper projection of $A$, and $\alpha$ is a ${ }^{*}$-isomorphism from $A$ onto $q A q$. If in addition $q A q$ is a full corner of $A$, then there is a cyclic exact sequence due to Paschke [49, Thm. 4.1]:

$$
\cdots \rightarrow K_{j}(A) \stackrel{\text { id }-\alpha_{*}}{\longrightarrow} K_{j}(A) \stackrel{i_{*}}{\longrightarrow} K_{j}\left(A \times_{\alpha} \mathbb{N}\right) \longrightarrow K_{1-j}(A) \stackrel{\text { id }-\alpha_{*}}{\longrightarrow} \cdots \quad(j=0,1) .
$$

The following appears in [12] for the case where $\sigma$ is a covering map and $G(X, \sigma)$ is minimal.

Proposition 9.1. Let $X$ be a compact metric space, and $\sigma: X \rightarrow X$ a surjective piecewise homeomorphism. Then there is an exact sequence

$$
\begin{array}{ccccc}
K_{0}\left(C^{*}(R(X, \sigma))\right) & \stackrel{\text { id }-\Phi_{*}}{\longrightarrow} & K_{0}\left(C^{*}(R(X, \sigma))\right) & \stackrel{i_{*}}{\longrightarrow} & K_{0}\left(C^{*}(X, \sigma)\right) \\
K_{1}\left(C^{*}(X, \sigma)\right) & \stackrel{i_{*}}{\longleftarrow} & K_{1}\left(C^{*}(R(X, \sigma))\right) & \stackrel{\text { id }-\Phi_{*}}{\longleftarrow} & K_{1}\left(C^{*}(R(X, \sigma))\right)
\end{array}
$$

where $\Phi$ is the canonical endomorphism of $C^{*}(R(X, \sigma))$; cf. (1).

Proof. If $\sigma$ is injective, then $C^{*}(R(X, \sigma))=C(X)$, and $\Phi$ is a *automorphism of $C(X)$. Then $C^{*}(X, \sigma) \cong C(X) \times_{\Phi} \mathbb{Z}$, and (41) is the Pimsner-Voiculescu exact sequence [52. If $\sigma$ is not injective, and $q=\Phi(1)$, then $q \neq 1$, and $\Phi$ is an isomorphism from $C^{*}(R(X, \sigma))$ onto the proper corner $q C^{*}(R(X, \sigma)) q$. Then (4) follows from Paschke's exact sequence (3) if we show that this corner is full, i.e., that the ideal it generates is all of $C^{*}(R(X, \sigma))$. For each $Y$ in Corollary 6.3, let $E_{Y}=\frac{1}{k(Y)} \sum_{i j} e_{i j}^{Y}$. Then $\Phi(1)=\sum_{Y} E_{Y}$, and so the ideal generated by $q=\Phi(1) \in C^{*}\left(R_{2}\right)$ is all of $C^{*}\left(R_{2}\right)$; in particular that ideal contains 1. Therefore $q C^{*}(R(X, \sigma)) q$ is a full corner of $C^{*}(R(X, \sigma))$, which completes the proof of the proposition.

Corollary 9.2. If $\tau: I \rightarrow I$ is piecewise monotonic and surjective, there is an exact sequence

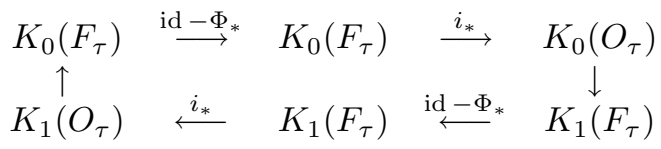

where $\Phi$ is the canonical endomorphism of $F_{\tau}$; cf. (11).

Proof. This follows from Proposition 9.1 and the definitions of $F_{\tau}$ and $O_{\tau}$.

Proposition 9.3. Let $G$ be an ordered abelian group and $T: G \rightarrow G$ a positive homomorphism. Let $G^{\infty}=\lim (T: G \rightarrow G)$ be the inductive limit, with canonical homomorphisms $\mu_{k}$ from the $\vec{k}$-th copy of $G$ into $G^{\infty}$. Then there is a unique order automorphism $T^{\infty}$ of $G^{\infty}$ such that $T^{\infty}\left(\mu_{k}(z)\right)=\mu_{k}(T z)$ for all $k$, and

$$
\begin{aligned}
\operatorname{coker}\left(\mathrm{id}-T^{\infty}\right) & \cong \operatorname{coker}(\mathrm{id}-T), \\
\operatorname{ker}\left(\mathrm{id}-T^{\infty}\right) & \cong \operatorname{ker}(\mathrm{id}-T) .
\end{aligned}
$$

Proof. The proof for the case $G=\mathbb{Z}^{n}$ in [9, Prop. 3.1] works for general $G$. 
Proposition 9.4. Let $\tau: I \rightarrow I$ be a piecewise monotonic surjective map, with associated local homeomorphism $\sigma: X \rightarrow X$. Let $\Phi$ be the canonical endomorphism of $F_{\tau}$, and $\mathcal{L}: C(X, \mathbb{Z}) \rightarrow C(X, \mathbb{Z})$ the transfer map. Then

$$
\begin{gathered}
K_{0}\left(O_{\tau}\right) \cong \operatorname{coker}\left(\mathrm{id}-\Phi_{*}\right) \cong \operatorname{coker}\left(\mathrm{id}-\mathcal{L}_{*}\right) \cong \operatorname{coker}(\mathrm{id}-\mathcal{L}), \\
K_{1}\left(O_{\tau}\right) \cong \operatorname{ker}\left(\mathrm{id}-\Phi_{*}\right) \cong \operatorname{ker}\left(\mathrm{id}-\mathcal{L}_{*}\right) \cong \operatorname{ker}(\mathrm{id}-\mathcal{L}) .
\end{gathered}
$$

Proof. We apply the exact sequence (5). Since $F_{\tau}$ is an AI-algebra, then $K_{1}\left(F_{\tau}\right)=$ 0 , and the first isomorphisms of (8) and (9) follow. Under the isomorphism of $K_{0}\left(F_{\tau}\right)$ with $D G(\tau), \Phi_{*}$ is carried to $\mathcal{L}_{*}^{-1}$ (Corollary (7.10), so $\left(\mathrm{id}-\Phi_{*}\right) K_{0}\left(F_{\tau}\right)$ is carried to

$$
\left(\mathrm{id}-\mathcal{L}_{*}^{-1}\right) D G(\tau)=\left(\mathrm{id}-\mathcal{L}_{*}\right) \mathcal{L}_{*}^{-1} D G(\tau)=\left(\mathrm{id}-\mathcal{L}_{*}\right) D G(\tau) .
$$

From this, the second isomorphism in (8) follows, and the second isomorphism of (9) follows by a similar argument.

By Proposition 7.5 and Definition [7.3, $D G(\tau)$ is isomorphic to the inductive $\operatorname{limit} \lim \left(\mathcal{L}: \mathcal{L}^{n}(C(X, \mathbb{Z})) \rightarrow \mathcal{L}^{n+1}(C(X, \mathbb{Z}))\right)$. Since $\tau$ (and therefore $\sigma$ and $\mathcal{L}$ ) is surjective, this is the same as the inductive $\operatorname{limit} \lim (\mathcal{L}: C(X, \mathbb{Z}) \rightarrow C(X, \mathbb{Z}))$. The induced map $\mathcal{L}^{\infty}$ on $D G(\tau)$ is $\mathcal{L}_{*}$; cf. Proposition 9.3 . Thus by (6)

$$
\operatorname{coker}\left(\mathrm{id}-\mathcal{L}_{*}\right) \cong \operatorname{coker}(\mathrm{id}-\mathcal{L}) \text {. }
$$

This completes the proof of the third isomorphism in (8). In a similar manner, from (7) we get the third isomorphism of (9).

Applying a result of Anantharaman-Delaroche [1, Renault [59, Prop. 2.6] has shown that for an essentially free local homeomorphism $\sigma: X \rightarrow X$, if for every non-empty open set $U \subset X$ there exists an open set $V \subset U$ and $m, n \in \mathbb{N}$ such that $\sigma^{n}(V)$ is properly contained in $\sigma^{m}(V)$, then $C^{*}(X, \sigma)$ is purely infinite. We apply this to get a sufficient condition for $O_{\tau}$ to be purely infinite.

Theorem 9.5. If $\tau: I \rightarrow I$ is piecewise monotonic, transitive, and not essentially injective, then $O_{\tau}$ is separable, simple, purely infinite, and nuclear, and is in the UCT class $\mathcal{N}$.

Proof. Separability follows from the fact that $F_{\tau}$ is an AF-algebra (Proposition 6.10), and the fact that $O_{\tau}$ is the crossed product of $F_{\tau}$ by an endomorphism. Simplicity follows from Proposition 5.3. Nuclearity of $C^{*}(X, \sigma)$ is established in 11, or see [59, Prop. 2.4]. Furthermore, $G(X, \sigma)$ is amenable [59, Prop. 2.4], so the full and reduced $\mathrm{C}^{*}$-algebras coincide. Since $\tau$ is transitive, then $\sigma$ is strongly transitive (Proposition 2.8), so as shown in the proof of Proposition 4.3, $\sigma$ is essentially free. We would like to show that for each open set $W$ there is an open set $V \subset W$ and positive integers $m, n$ such that $\sigma^{m}(V)$ is properly contained in $\sigma^{n}(V)$. By Theorem 6.12, there is a partition $X_{1}, \ldots, X_{p}$ of $X$ into clopen sets invariant under $\sigma^{p}$, such that $\sigma^{p}$ is topologically exact on each $X_{i}$. Choose $X_{j}$ so that $X_{j} \cap W \neq \emptyset$, and let $V$ be a proper open subset of $X_{j} \cap W$. By exactness of $\sigma^{p}$ on $X_{j}$, there exists $k \geq 0$ such that $\left(\sigma^{p}\right)^{k} V=X_{j} \supset V$, which proves that $O_{\tau}=C^{*}(X, \sigma)$ is purely infinite. Since AI-algebras are in the UCT class $\mathcal{N}$, and that class is closed under crossed products by $\mathbb{N}$, then $O_{\tau}$ is in $\mathcal{N}$.

The hypothesis that $\tau$ is not essentially injective cannot be omitted in Theorem 9.5. For example, if $\tau: I \rightarrow I$ is the piecewise linear map given by $x \mapsto x+\theta \bmod 1$, 
with $\theta$ irrational, then $\tau$ is transitive and essentially injective. The associated local homeomorphism $\sigma: X \rightarrow X$ is a homeomorphism, and $O_{\tau} \cong C(X) \times_{\Phi} \mathbb{Z}$. Here Lebesgue measure induces a measure on $X$, invariant with respect to $\sigma$, so $O_{\tau}$ has a tracial state. Since $O_{\tau}$ is simple (Proposition 5.2), this tracial state must be faithful, so $O_{\tau}$ cannot be purely infinite.

For later reference, we recall the classification results of Kirchberg and Phillips ([33] and [50]). For an exposition, see the book of Rørdam [61, §8.4].

Theorem 9.6 (Kirchberg and Phillips). Let $A_{1}$ and $A_{2}$ be unital $C^{*}$-algebras which are separable, simple, purely infinite, and nuclear, and are in the UCT class $\mathcal{N}$. If there is a unital isomorphism of $K_{0}\left(A_{1}\right)$ onto $K_{0}\left(A_{2}\right)$, and an isomorphism of $K_{1}\left(A_{1}\right)$ onto $K_{1}\left(A_{2}\right)$, then $A_{1}$ and $A_{2}$ are isomorphic.

By Theorems 9.5 and 9.6, when $\tau$ is piecewise monotonic, transitive, and not essentially injective, the $\mathrm{C}^{*}$-algebra $O_{\tau}$ is determined by $K_{0}\left(O_{\tau}\right)$ and $K_{1}\left(O_{\tau}\right)$.

\section{TRACES AND KMS States}

We recall the following definition from 64 .

Definition 10.1. Let $X$ be a compact metric space, $\psi: X \rightarrow X$ a map that takes Borel sets to Borel sets, and $m$ a probability measure on $X$. Then $\psi$ scales $m$ by $a$ factor $s$ if $m(\psi(E))=s m(E)$ for all Borel sets $E$ on which $\psi$ is $1-1$.

Lemma 10.2. Let $\tau: I \rightarrow I$ be piecewise monotonic, $\sigma: X \rightarrow X$ the associated local homeomorphism. Let $m$ be a measure on $X$ scaled by $\sigma$ by a factor $s$. Then there is a trace on $F_{\tau}=C^{*}(R(X, \sigma))$ which satisfies

$$
\operatorname{tr}(f)=\int_{X} f(x, x) d m \text { for } n \in \mathbb{N} \text { and } f \in C^{*}\left(R_{n}\right),
$$

and this trace is uniquely determined by (10).

Proof. By Lemma 6.2, we can construct a partition of $X$ into clopen sets each of which $\sigma^{n}$ is injective, and whose images are either equal or disjoint. Let $Y$ be one of these images, and $E_{1}, \ldots, E_{q}$ a partition of $\sigma^{-n} Y$ into clopen sets mapped bijectively by $\sigma^{n}$ onto $Y$. Let $\left\{E_{i j}\right\}$ be the canonical matrix units in Proposition6.1, i.e., $E_{i j}(x, y)=1$ if $x \in E_{i}$ and $y \in E_{j}$, and $\sigma^{n} x=\sigma^{n} y$. Define $\operatorname{tr}$ on $\bigcup_{n} C^{*}\left(R_{n}\right)$ by (10). By Proposition 6.1 and Corollary 6.3. to prove that $\operatorname{tr}(f g)=\operatorname{tr}(g f)$ for all $f, g \in C^{*}\left(R_{n}\right)$, it suffices to show that $\operatorname{tr}(e f)=\operatorname{tr}(f e)$ for matrix units $f, e \in\left\{E_{i j}\right\}$. Note that $\operatorname{tr}\left(E_{i j}\right)=0$ if $i \neq j$. Thus $\operatorname{tr}\left(E_{i j} E_{i^{\prime} j^{\prime}}\right) \neq 0$ only if $i=j^{\prime}$ and $j=i^{\prime}$, i.e., $E_{i^{\prime} j^{\prime}}=E_{j i}$. Thus we need to verify that $\operatorname{tr}\left(E_{i j} E_{j i}\right)=\operatorname{tr}\left(E_{j i} E_{i j}\right)$, i.e., that $\operatorname{tr}\left(E_{i i}\right)=\operatorname{tr}\left(E_{j j}\right)$. We have $\operatorname{tr}\left(E_{i i}\right)=m\left(E_{i}\right)$, so the key requirement is that $m\left(E_{i}\right)=m\left(E_{j}\right)$ for all $i, j$. Since $\sigma^{n}$ maps $E_{i}$ and $E_{j}$ bijectively onto $Y$, and $m$ is scaled by $\sigma$, then $m(Y)=s^{n} m\left(E_{i}\right)=s^{n} m\left(E_{j}\right)$. This completes the proof that tr defines a trace on each $C^{*}\left(R_{n}\right)$. It is clear that $\operatorname{tr}$ is positive and $\operatorname{tr}(1)=1$, so tr has norm one on each $C^{*}\left(R_{n}\right)$, and therefore extends uniquely to a trace on $C^{*}(R(X, \sigma))$.

We now describe one way to find scaling measures.

Definition 10.3. A map $\tau: I \rightarrow I$ is uniformly piecewise linear if there is a number $s>0$, and a partition $0=a_{0}<a_{1}<\cdots<a_{n}=1$, such that $\tau$ is linear with slope $\pm s$ on each interval $\left(a_{i}, a_{i+1}\right)$. 
Note that if $\tau: I \rightarrow I$ is uniformly piecewise linear with slopes $\pm s$, then Lebesgue measure $m$ is scaled by $\tau$ by the factor $s$. Let $\sigma: X \rightarrow X$ be the associated local homeomorphism, and define a measure $\mu$ on $X$ by $\mu(A)=m\left(\pi\left(A \cap X_{0}\right)\right)$. Then it is readily verified that $\mu$ is a probability measure on $X$ scaled by $\sigma$ by a factor $s$ (cf. 64, Prop. 3.3]), and so induces a trace on $F_{\tau}=C^{*}(R(X, \sigma))$ satisfying (10) (with $\mu$ in place of $m$ ).

Uniformly piecewise linear maps occur more frequently than might be thought. In the following result, $\tau$ is assumed to be right continuous at 0 , left continuous at 1 , and either left or right continuous at any other points of discontinuity. Recall that the topological entropy of $\tau$ and $\sigma$ are the same (Proposition 2.8).

Proposition 10.4 ([4] $)$. If $\tau: I \rightarrow I$ is piecewise monotonic and transitive, then $\tau$ is conjugate to a uniformly piecewise linear map with slopes $\pm s$, where $s>1$. The scaling factor $s$ equals $e^{h_{\tau}}$.

Proof. The conjugacy result is in [46 and 64, Cor. 4.4]. If $\tau$ is continuous, then so is the conjugate uniformly piecewise linear map, and the fact that $\ln s=h_{\tau}$ follows from [42. For $\tau$ discontinuous, see [64, Cor. 4.4].

Theorem 10.5. Let $\tau: I \rightarrow I$ be piecewise monotonic, not essentially injective, and transitive, and let $F_{\tau}=\bigoplus A_{i}$ be the decomposition as a sum of simple AFalgebras given in Corollary 6.13. Then each $A_{i}$ has a unique tracial state $\tau_{i}$. There is a tracial state on $F_{\tau}$ scaled by $\Phi$, and such a tracial state is unique. The scaling factor is $e^{-h_{\tau}}$.

Proof. Let $\sigma: X \rightarrow X$ be the associated local homeomorphism. Let $X_{1}, \ldots, X_{n}$ be a partition of $X$ into clopen sets, cyclically permuted by $\sigma$, such that $\sigma^{n}$ restricted to each $X_{i}$ is topologically exact; cf. Theorem 6.12.

There is a unique state on $D G(\tau) \cong K_{0}\left(F_{\tau}\right)$ scaled by $\mathcal{L}_{*}$ by the factor $s=$ $\exp \left(h_{\tau}\right)$ (64, Thm. 5.3]). Since there is a bijection of tracial states on a unital AFalgebra and states on the associated dimension group (cf., e.g., 61, Prop. 1.5.5]), and since $\Phi_{*}=\mathcal{L}_{*}^{-1}$, it follows that there is a unique tracial state on $F_{\tau}$ scaled by $\Phi$ by the factor $1 / s=\exp \left(-h_{\tau}\right)$. Furthermore, by Theorem 7.9 , $K_{0}\left(A_{i}\right)=$ $K_{0}\left(C^{*}\left(R\left(X_{i}, \sigma^{n}\right)\right)\right)$ is isomorphic to $G_{\left.\sigma^{n}\right|_{X_{i}}}$, which has a unique state ([64, Thm. 5.3]). It follows that $A_{i}$ has a unique tracial state.

The uniqueness statement regarding the scaled trace in Theorem 10.5 can fail if $\tau$ is essentially injective. For example, there are minimal interval exchange maps with more than one invariant measure; cf. 31] or 32. Each invariant measure induces a trace on $F_{\tau}$ scaled by $\Phi$ by the factor $s=1$.

Corollary 10.6. If $\tau:[0,1] \rightarrow[0,1]$ is piecewise monotonic and is topologically exact, then $F_{\tau}$ is simple and has a unique tracial state. The canonical endomorphism $\Phi$ of $F_{\tau}$ scales the trace by a factor $e^{-h_{\tau}}$, where $h_{\tau}$ is the topological entropy of $\tau$.

Proof. Topological exactness of $\tau$ implies topological exactness of $\sigma$, so there is just one summand in the decomposition $F_{\tau}=\bigoplus A_{i}$ in Theorem 10.5.

We turn to the question of KMS states on the algebra $O_{\tau}$. Suppose that $\tau: I \rightarrow I$ is surjective, so that $O_{\tau}$ is isomorphic to $F_{\tau} \times_{\Phi} \mathbb{N}$. Then $O_{\tau}$ is the universal $\mathrm{C}^{*}{ }_{-}$ algebra generated by $F_{\tau}$ and an isometry $v$ such that $v a v^{*}=\Phi(a)$ for $a \in F_{\tau}$. For each scalar $\lambda$ with $|\lambda|=1$, there is a unique ${ }^{*}$-automorphism $\alpha_{\lambda}$ of $O_{\tau}$ which 
fixes $F_{\tau}$ and takes $v$ to $\lambda v$. This gives an action of $\mathbb{T}$ on $O_{\tau}$, or alternatively, an action of $\mathbb{R}$ (by taking $\lambda=e^{i t}$ ). With respect to this action, the set of fixed points is $F_{\tau}$, and there is a faithful conditional expectation from $O_{\tau}$ onto $F_{\tau}$ given by $E(b)=\int_{\mathbb{T}} \alpha_{\lambda}(b) d \lambda$. See [48, 49].

Theorem 10.7. If $\tau: I \rightarrow I$ is transitive and is not essentially injective, then $O_{\tau}$ has a unique $\beta$-KMS state for $\beta=h_{\tau}$, and there is no other $\beta$-KMS state for $0 \leq \beta<\infty$.

Proof. In the remarks at the end of [48, it is observed that $\beta$-KMS states for $0<\beta<\infty$ are precisely those of the form $\operatorname{tr} \circ E$, where tr is a trace scaled by $\Phi$ by the factor $e^{-\beta}$, and $E$ is the conditional expectation from $O_{\tau}$ onto $F_{\tau}$ described above. We know $F_{\tau}$ has a unique trace scaled by $\Phi$, and that the scaling factor is $\exp \left(-h_{\tau}\right)$, so the desired result follows for $0<\beta$. For the case $\beta=0$, a KMS state is a tracial state on $O_{\tau}$. Since the latter is simple and purely infinite (Theorem 9.5), no such tracial state exists, so no $\beta$-KMS state exists for $\beta=0$.

If $\sigma$ is exact and positively expansive, the results on KMS states in Theorem 10.7 follow from [39, Thm. 3.5]. However, the map $\sigma$ will not be positively expansive unless the forward orbit under $\tau$ of the set $C$ of endpoints of intervals of monotonicity is finite, i.e., $\tau$ is Markov (as defined below). (For details, see 64, remark after Thm. 4.5].)

\section{MARKOV MAPS}

In the remaining sections, we will apply the results developed so far to particular families of interval maps. In Figure 1, some of the examples that will be discussed are portrayed.
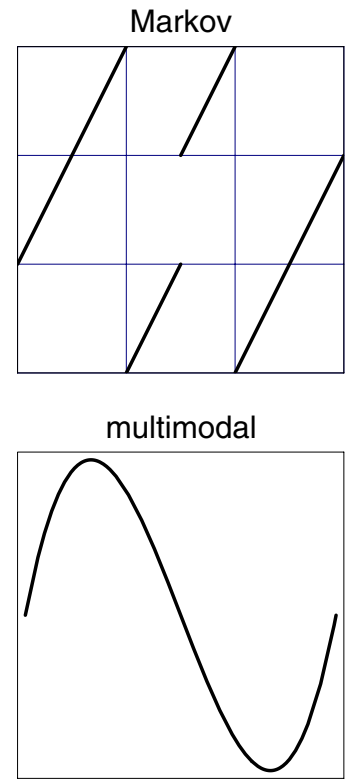
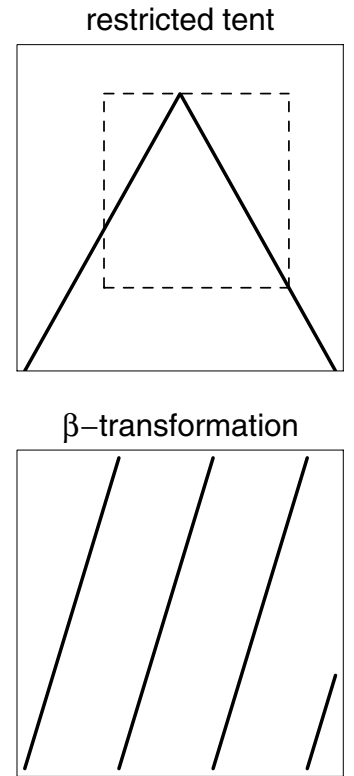
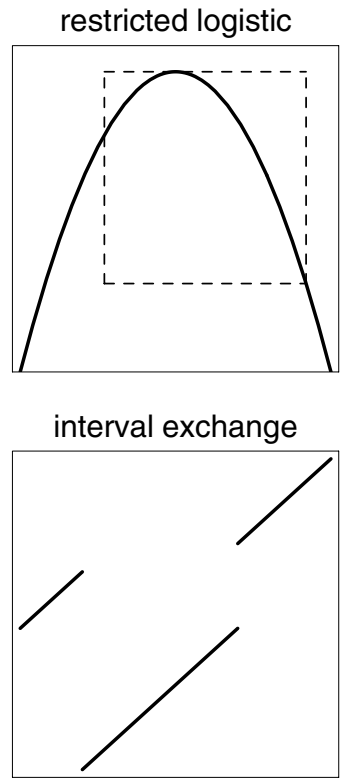

Figure 1. Examples of piecewise monotonic maps 
Recall that if $\tau: I \rightarrow I$ is piecewise monotonic, with associated partition $C$, and is discontinuous at $c \in C$, we view $\tau$ as multivalued at $c$, with the values given by the left and right limits of $\tau$. For $A \subset[0,1]$, we write $\widehat{\tau}(A)$ to denote the set of values of $\tau$ at points in $A$, including the possible multiple values at points in $C \cap A$.

Definition 11.1. Let $\tau: I \rightarrow I$ be piecewise monotonic. A Markov partition for $\tau$ is a partition $0=b_{0}<b_{1}<\cdots<b_{n}=1$, with each $b_{i}$ being in $I_{1}$, such that for each $i, \tau$ is monotonic on $\left(b_{i}, b_{i+1}\right)$, such that $\overline{\tau\left(b_{i}, b_{i+1}\right)}$ is a union of intervals of the form $\left[b_{j}, b_{j+1}\right]$, and such that for some $n \geq 0, \widehat{\tau}^{n}(C) \subset\left\{b_{0}, b_{1}, \ldots, b_{n}\right\}$. We say $\tau$ is Markov if it admits a Markov partition, The incidence matrix for $\tau$ with respect to this Markov partition is the zero-one $n \times n$ matrix $A$ where $A_{i j}=1$ iff $\tau\left(b_{i-1}, b_{i}\right) \supset\left(b_{j-1}, b_{j}\right)$.

If $\tau$ is piecewise monotonic with associated partition $C$ and is Markov, then one possible Markov partition is the set of points in the forward orbit of $C$. This will be the most common kind of Markov partition that we use, but the slightly greater generality allowed in the definition above will be useful in showing that all CuntzKrieger algebras $O_{A}$ arise as $O_{\tau}$ for some piecewise monotonic map $\tau$; cf. Corollary 11.9 .

If $\sigma: X \rightarrow X$ is the associated local homeomorphism, then the order intervals $I\left(b_{i-1}, b_{i}\right)$ will form a partition of $X$ such that each $\sigma\left(I\left(b_{i-1}, b_{i}\right)\right)$ is a union of some order intervals of the form $I\left(b_{j-1}, b_{j}\right)$. Since the range of $\tau$ or $\sigma$ will be a union of partition intervals, it follows that both $\tau$ and $\sigma$ will be eventually surjective.

Definition 11.2. If $A$ is an $n \times n$ zero-one matrix, $G_{A}$ denotes the stationary inductive limit $\mathbb{Z}^{n} \stackrel{A}{\longrightarrow} \mathbb{Z}^{n}$ in the category of ordered abelian groups. Here $A$ acts by right multiplication, and $G_{A}$ will be a dimension group. The action of $A$ induces an automorphism of $G_{A}$ denoted $A_{*}$.

Proposition 11.3. If $\tau: I \rightarrow I$ is piecewise monotonic and Markov, with incidence matrix $A$, then $K_{0}\left(F_{\tau}\right) \cong G_{A}$. If $\Phi$ is the canonical *-automorphism of $F_{\tau}$, then the induced automorphism $\mathcal{L}_{*}=\left(\Phi_{*}\right)^{-1}$ on $K_{0}\left(F_{\tau}\right)$ is carried to $A_{*}$.

Proof. This follows from the identification of $K_{0}\left(F_{\tau}\right)$ with $D G(\tau)$ (Corollary 7.10$)$, and the isomorphism of $\left(D G(\tau), \mathcal{L}_{*}\right)$ with $\left(G_{A}, A_{*}\right)$ [63, Prop. 8.4].

Corollary 11.4. If $\tau: I \rightarrow I$ is piecewise monotonic, surjective, and Markov, with incidence matrix $A$, then

$$
\begin{gathered}
K_{0}\left(O_{\tau}\right) \cong \operatorname{coker}(\mathrm{id}-A), \\
K_{1}\left(O_{\tau}\right) \cong \operatorname{ker}(\mathrm{id}-A) .
\end{gathered}
$$

Proof. By Propositions 9.4 and 11.3 .

$$
K_{0}\left(O_{\tau}\right) \cong \operatorname{coker}\left(\mathrm{id}-\mathcal{L}_{*}\right) \cong \operatorname{coker}\left(\mathrm{id}-A_{*}\right),
$$

which is isomorphic to coker(id $-A$ ) by Proposition 9.3 The second isomorphism in the statement of the corollary follows in a similar fashion.

If a piecewise monotonic Markov map $\tau$ is not surjective, then the eventual range $Y$ of $\sigma$ will be a union of some of the order intervals in the Markov partition. Restricting $\sigma$ to its eventual range gives a Markov map whose incidence matrix $A_{Y}$ will be the restriction of the original incidence matrix to the rows 
and columns corresponding to intervals in the eventual range. The corresponding algebras $C^{*}\left(R\left(Y,\left.\sigma\right|_{Y}\right)\right)$ and $C^{*}\left(Y,\left.\sigma\right|_{Y}\right)$ will be strongly Morita equivalent to $F_{\tau}=C^{*}(R(X, \sigma))$ and $O_{\tau}=C^{*}(X, \sigma)$, respectively, as remarked at the end of Section 3 Morita equivalent $\mathrm{C}^{*}$-algebras have the same K-groups, so the isomorphisms in Corollary 11.4 will hold with $A_{Y}$ in place of $A$.

Let $X_{A}$ be the set of all sequences in $\{1,2, \ldots, n\}^{\mathbb{N}}$ such that $i$ is followed by $j$ only if $A_{i j}=1$, and let $\sigma_{A}$ be the one-sided shift map on $X_{A}$. We are going to characterize when $(X, \sigma)$ is conjugate to $\left(X_{A}, \sigma_{A}\right)$.

For each $n \times n$ zero-one matrix $A$ satisfying a certain "Condition I", Cuntz and Krieger 10 defined an AF-algebra $F_{A}$ and an algebra $O_{A}$. For finite directed graphs, Condition I is equivalent to the following condition of KumjianPask-Raeburn [38, Lemma 3.3]. (For a directed graph we will always require that there are no multiple edges between vertices.)

Definition 11.5. A directed graph satisfies Condition $L$ if for each cycle $v_{1} v_{2}$ $\cdots v_{n} v_{1}$, there is an index $i$ and an edge from $v_{i}$ to a vertex other than $v_{i+1} \bmod n$. If $A$ is a zero-one $n \times n$ matrix, $A$ satisfies Condition L if the associated directed graph satisfies Condition L.

Definition 11.6. Let $\tau: I \rightarrow I$ be piecewise monotonic and Markov, with associated local homeomorphism $\sigma: X \rightarrow X$. Let $E_{1}, \ldots, E_{n}$ be the associated Markov partition for $\sigma: X \rightarrow X$, with incidence matrix $A$. The itinerary map $S: X \rightarrow X_{A}$ is given by $S(x)=s_{0} s_{1} s_{2} \cdots$, where $\sigma^{k}(x) \in E_{s_{k}}$. We say that itineraries separate points of $X$ if the itinerary map is 1-1. (This is independent of the choice of Markov partition.)

We now describe when itineraries separate points. Recall that an interval $J$ is a homterval for a piecewise monotonic map $\tau: I \rightarrow I$ if $\tau^{n}$ is a homeomorphism on $J$ for all $n$. A piecewise monotonic Markov map $\tau: I \rightarrow I$ is piecewise linear if for each interval $J$ of the Markov partition, there is a partition of $J$ into subintervals on whose interior $\tau$ is linear. We allow $\tau$ to be discontinuous at finitely many points in each Markov partition interval, but require that its slope be constant within each Markov partition interval. (See the Markov map in Figure 1 for an example.)

Proposition 11.7. Let $\tau: I \rightarrow I$ be piecewise monotonic and Markov, with incidence matrix A. These are equivalent:

(1) $\tau$ has no homtervals.

(2) Itineraries separate points of $X$.

(3) $(X, \sigma)$ is conjugate to the one-sided shift of finite type $\left(X_{A}, \sigma_{A}\right)$.

If $\tau$ is also piecewise linear, these conditions are equivalent to A satisfying Condition $L$.

Proof. [63, Props. 8.5 and 8.8]

Proposition 11.8. Let $\tau: I \rightarrow I$ be piecewise monotonic and Markov, and let $\sigma: X \rightarrow X$ be the associated local homeomorphism. If $A$ is the associated incidence matrix, and if itineraries separate points of $X$, then $F_{\tau} \cong F_{A}$ and $O_{\tau} \cong O_{A}$.

Proof. By Proposition 11.7 $(X, \sigma)$ is conjugate to $\left(X_{A}, \sigma_{A}\right)$. Thus $C^{*}(R(X, \sigma)) \cong$ $C^{*}\left(R\left(X_{A}, \sigma_{A}\right)\right)$, and $C^{*}\left(R\left(X_{A}, \sigma_{A}\right)\right) \cong F_{A}$ by (12, Example 2]). Hence $F_{\tau} \cong F_{A}$. In a similar fashion, since $C^{*}\left(X_{A}, \sigma_{A}\right) \cong O_{A}$ [59, p. 10], then $O_{\tau} \cong O_{A}$. 
Corollary 11.9. For each $n \times n$ zero-one matrix A satisfying Cuntz-Krieger's Condition I, there is a piecewise linear Markov map $\tau$ such that $F_{\tau} \cong F_{A}$ and $O_{\tau} \cong O_{A}$.

Proof. Let $\tau$ be any piecewise linear Markov map, with a Markov partition whose associated incidence matrix is $A$. Since Condition $\mathrm{I}$ is equivalent to Condition $\mathrm{L}$ [38. Lemma 3.3], the corollary follows from Propositions 11.7 and 11.8.

If $\tau$ is piecewise linear, Markov, and surjective, with incidence matrix $A$, we can determine simplicity of $F_{\tau}$ and $O_{\tau}$ from the matrix $A$. Indeed, $\tau$ is topologically exact iff $A$ is primitive (63, Cor. 8.9]), and this is equivalent to $F_{\tau}$ being simple (Proposition 5.2). Similarly, $\tau$ is transitive iff $A$ is irreducible and is not a permutation matrix, and this is equivalent to $O_{\tau}$ being simple (Proposition 5.3).

The next two results will be used later for Markov maps that are unimodal or are $\beta$-transformations. We write $\mathbb{Z}[t] I(0,1)$ for the set of elements in $C(X, \mathbb{Z})$ of the form $p(\mathcal{L}) I(0,1)$, with $p$ a polynomial with integral coefficients.

Lemma 11.10. Let $L$ be an endomorphism of an abelian group $G$, and $M$ a subgroup of $G$ invariant under $L$, such that for each $g \in G$ there exists $k \in \mathbb{N}$ such that $L^{k} g \in M$. Then

$$
\operatorname{ker}\left(\left.(\mathrm{id}-L)\right|_{M}\right)=\operatorname{ker}(\mathrm{id}-L) \text { and } \operatorname{coker}\left(\left.(\mathrm{id}-L)\right|_{M}\right) \cong \operatorname{coker}(\mathrm{id}-L) .
$$

Proof. Suppose first that $g \in \operatorname{ker}(\mathrm{id}-L)$, so that $L g=g$. Choose $k$ so that $L^{k} g \in$ $M$. Then $g=L^{k} g \in M$, so $g \in \operatorname{ker}\left(\left.(\mathrm{id}-L)\right|_{M}\right)$. Thus $\operatorname{ker}\left(\left.(\mathrm{id}-L)\right|_{M}\right)=\operatorname{ker}(\mathrm{id}-L)$.

Next define $\phi: \operatorname{coker}\left(\left.(\mathrm{id}-L)\right|_{M}\right) \rightarrow \operatorname{coker}(\mathrm{id}-L)$ by

$$
\phi(m+(\mathrm{id}-L) M)=m+(\mathrm{id}-L) G .
$$

If $m \in($ id $-L) M$, then $m \in(\mathrm{id}-L) G$, so $\phi$ is well defined. If $m \in M$ and $\phi(m)=0$, choose $g \in G$ so that $m=(\mathrm{id}-L) g$. Now choose $k \in \mathbb{N}$ so that $L^{k} g \in M$. Then $L^{k} m=L^{k}(g-L g)=(\mathrm{id}-L) L^{k} g \in(\mathrm{id}-L) M$. Note that

$$
m-L^{k} m=(\mathrm{id}-L) \sum_{i=0}^{k-1} L^{i} m \in(\mathrm{id}-L) M .
$$

Thus $m=L^{k} m+\left(m-L^{k} m\right) \in($ id $-L) M$, so $\phi$ is injective. To see that $\phi$ is surjective, let $g \in G$, and again choose $k \in \mathbb{N}$ such that $L^{k} g \in M$. Then

$$
\phi\left(L^{k} g\right)=L^{k} g+(\mathrm{id}-L) G=g+\left(L^{k} g-g\right)+(\mathrm{id}-L) G=g+(\mathrm{id}-L) G .
$$

Thus $\phi$ is surjective, which completes the proof.

Recall that a module is cyclic if it is generated by a single element, called a cyclic element. If $M$ is a subgroup of $C(X, \mathbb{Z})$, invariant under the transfer map $\mathcal{L}$, and $p \in \mathbb{Z}[t]$ is a monic polynomial such that $p(\mathcal{L}) M=0$, we say $p$ is the minimal polynomial for $\mathcal{L}$ on $M$ if no polynomial of lower degree annihilates $\left.\mathcal{L}\right|_{M}$. Note that it may happen that no polynomial annihilates $\left.\mathcal{L}\right|_{M}$, so that there is no minimal polynomial for $\mathcal{L}$ on $M$.

Proposition 11.11. Let $\tau: I \rightarrow I$ be piecewise monotonic and surjective. Assume that $I(0,1)$ is a cyclic element for the module $D G(\tau)$, and that the minimal polynomial for $\mathcal{L}$ on $M=\mathbb{Z}[t] I(0,1) \subset C(X, \mathbb{Z})$ is

$$
\begin{gathered}
m(\lambda)=\lambda^{p}-b_{0} \lambda^{p-1}-b_{1} \lambda^{p-2}-\cdots-b_{p-1} . \\
\text { Let } n=|m(1)|=\left|1-\left(b_{0}+b_{1}+\cdots+b_{p-1}\right)\right| .
\end{gathered}
$$


If $n \neq 0$, then

$$
K_{0}\left(O_{\tau}\right) \cong \mathbb{Z} / n \mathbb{Z} \text { and } K_{1}\left(O_{\tau}\right) \cong 0,
$$

and if in addition $\tau$ is transitive, then $O_{\tau} \cong O_{n+1}$.

If $n=0$, then

$$
K_{0}\left(O_{\tau}\right) \cong \mathbb{Z} \text { and } K_{1}\left(O_{\tau}\right) \cong \mathbb{Z}
$$

Regardless of the value of $n,[1]_{0}$ is a generator of $K_{0}\left(O_{\tau}\right)$.

Proof. We first show that

$$
f \in C(X, \mathbb{Z}) \Longrightarrow \exists k \in \mathbb{N} \text { such that } \mathcal{L}^{k} f \in M .
$$

Since $I(0,1)$ is cyclic for $\mathcal{L}_{*}$, there exists a Laurent polynomial $q$ with integer coefficients such that $[f]=q\left(\mathcal{L}_{*}\right) I(0,1)$. Choose $j \in \mathbb{N}$ such that $t^{j} q(t) \in \mathbb{Z}[t]$. Then $\left[\mathcal{L}^{j} f\right]=\mathcal{L}_{*}^{j} q\left(\mathcal{L}_{*}\right) I(0,1)$, so for some $m \in \mathbb{N}, \mathcal{L}^{j+m} f=\mathcal{L}^{m+j} q(\mathcal{L}) I(0,1) \in M$. Thus (12) holds.

Let $V$ be the rational linear span of $M$ in $C(X, \mathbb{R})$. Since $v_{0}=I(0,1)$ is cyclic for $\mathcal{L}$ on $M$, then for each polynomial $g \in \mathbb{Z}[t]$ of degree less than $p, g(\mathcal{L}) v_{0} \neq$ 0 . (Otherwise $g\left(\left.\mathcal{L}\right|_{M}\right)=0$, contrary to the assumption that $m$ is the minimal polynomial.) Therefore, no rational linear combination of $v_{0}, \mathcal{L} v_{0}, \ldots, \mathcal{L}^{p-1} v_{0}$ is zero, so these form a basis of $V \cong \mathbb{Q}^{p}$, and $M$ consists of integral linear combinations of this basis, so $M \cong \mathbb{Z}^{p}$. For this basis, the matrix for $\left.\mathcal{L}\right|_{M}$ is

$$
B=\left(\begin{array}{ccccc}
0 & 1 & 0 & \cdots & 0 \\
0 & 0 & 1 & \cdots & 0 \\
0 & 0 & 0 & \cdots & 0 \\
\cdots & \cdots & \cdots & \cdots & \cdots \\
0 & 0 & 0 & \cdots & 1 \\
b_{0} & b_{1} & b_{2} & \cdots & b_{p-1}
\end{array}\right)
$$

where $B$ acts by right multiplication on $\mathbb{Q}^{p}$. Now by elementary operations over $\mathbb{Z}$ (i.e., operations that exchange rows, multiply a row by -1 , or add an integral multiple of one row to another, or the analogous column operations), the matrix (id $-B)$ can be transformed to a diagonal matrix $D$ with entries $(1,1, \ldots, 1, n)$, where $n=\left|1-\left(b_{0}+b_{1}+\cdots+b_{p-1}\right)\right|$. (This is the Smith form of the matrix $B$; cf. [43, p. 248] and [45]). These elementary operations can be carried out by left or right multiplication by invertible integral matrices $W_{1}$ and $W_{2}$, with $W_{1}^{-1}$ and $W_{2}^{-1}$ also having integer entries, so that $D=W_{1}(\mathrm{id}-B) W_{2}$. It follows that the kernels of $D$ and id $-B$, when viewed as operators on $\mathbb{Z}^{p}$ by right multiplication, are isomorphic, and similarly their cokernels are isomorphic. Thus if $n \neq 0$, then

$$
\begin{gathered}
\operatorname{coker}\left(\left.(\mathrm{id}-\mathcal{L})\right|_{M}\right) \cong \mathbb{Z}^{p} /\left(\mathbb{Z}^{p}(\mathrm{id}-B)\right) \cong \mathbb{Z}^{p} / \mathbb{Z}^{p} D \cong \mathbb{Z} / n \mathbb{Z}, \\
\operatorname{ker}\left(\left.(\mathrm{id}-\mathcal{L})\right|_{M}\right) \cong \operatorname{ker}(\mathrm{id}-B) \cong \operatorname{ker} D \cong 0 .
\end{gathered}
$$

If $n=0$, then

$$
\begin{gathered}
\operatorname{coker}\left(\left.(\mathrm{id}-\mathcal{L})\right|_{M}\right) \cong \mathbb{Z}^{p} /\left(\mathbb{Z}^{p} D\right) \cong \mathbb{Z}, \\
\operatorname{ker}\left(\left.(\operatorname{id}-\mathcal{L})\right|_{M}\right) \cong \operatorname{ker} D \cong \mathbb{Z} .
\end{gathered}
$$

Combining these results with Lemma 11.10 and Proposition 9.4 gives the K-groups described in the proposition.

Since $M=\mathbb{Z}[t] v_{0}$, every element $v$ of $M$ can be written in the form $p(\mathcal{L}) v_{0}$, and thus its image in the quotient $M /(\mathrm{id}-\mathcal{L}) M$ is the same as the image of $p(1) v_{0}$. Hence the image of $v_{0}=I(0,1)$ generates the quotient. The element of $K_{0}\left(F_{\tau}\right)$ 
corresponding to the identity in $F_{\tau}$ is $I(0,1)$, and the inclusion of $F_{\tau}$ in $O_{\tau}$ is unital, so $[1]_{0}$ generates $K_{0}\left(O_{\tau}\right)$.

Finally, suppose that $n \neq 0$ and that $\tau$ is transitive. We would like to apply Theorem [9.5. for that purpose, we need to know that $\tau$ is not essentially injective. If $\tau$ were essentially injective, then $\sigma$ would be injective (63, Lemma 11.3]). Since $\tau$ (and then $\sigma$ ) are surjective, then $\sigma$ would be a homeomorphism, so $\mathcal{L} I(0,1)=$ $I(0,1)$. Thus the minimal polynomial of $\mathcal{L}$ on $M=\mathbb{Z}[t] I(0,1)$ would be $m(t)=t-1$, so $m(1)=0$, contrary to assumption. Thus $\tau$ is not essentially injective, so by Theorem 9.5. $O_{\tau}$ is simple, separable, nuclear, purely infinite, and in the UCT class $\mathcal{N}$, so by the results of Kirchberg [33] and Phillips [50] (Theorem 9.6), is determined by its K-groups. By the isomorphisms in (11), the K-groups of $O_{\tau}$ coincide with those of $O_{n+1}$, so these algebras are isomorphic.

If $m(t)$ is the minimal polynomial for $\mathcal{L}$ on $M=\mathbb{Z}[t] I(0,1)$, and $p(t)$ is the minimal polynomial for the incidence matrix $A$, then $m$ and $p$ are closely related. In Proposition 11.11, let $E_{1}, \ldots, E_{n}$ be the intervals for a Markov partition of $\sigma$, and $A$ the incidence matrix. The action of $\mathcal{L}$ on $\sum_{i} z_{i} E_{i}$ for $z_{i} \in \mathbb{Z}$ is given by right multiplication by $A$ on $\left(z_{1}, z_{2}, \ldots, z_{n}\right)$. Since $I(0,1)=E_{1}+\cdots+E_{n}$, then $p(A)=0$ implies $p(\mathcal{L}) I(0,1)=0$, so $m(t)$ divides $p(t)$. On the other hand, since by hypothesis $I(0,1)$ is a cyclic element for $\mathcal{L}_{*}$, for each $i$ there exists $f_{i} \in \mathbb{Z}\left[t, t^{-1}\right]$ such that $f_{i}\left(\mathcal{L}_{*}\right) I(0,1)=\left[E_{i}\right]$. For a suitable $k \geq 0, f_{i}(t) t^{k} \in \mathbb{Z}[t]$, so for some $K \geq 0$, $\mathcal{L}^{K} E_{i} \in \mathbb{Z}[t] I(0,1)$ for all $i$. Then $m(\mathcal{L}) \mathcal{L}^{K} E_{i}=0$ for all $i$, so $m(A) A^{K}=0$. Hence $p(t)$ divides $m(t) t^{K}$, and thus $p(t)=t^{j} m(t)$ for some $j \geq 0$. Then $p(1)=m(1)$, so in Proposition 11.11, we could replace $m$ by $p$.

Example 11.12. Let $\tau$ be the tent map, i.e., $\tau(x)=2 x$ if $0 \leq x \leq 1 / 2$, and $\tau(x)=$ $2-2 x$ if $1 / 2 \leq x \leq 1$. Then $\tau$ is topologically exact, so $F_{\tau}$ is a simple AF-algebra and $O_{\tau}$ is simple and purely infinite. Furthermore, $\tau$ is Markov, with incidence matrix $\left(\begin{array}{ll}1 & 1 \\ 1 & 1\end{array}\right)$, and the inductive limit of $\mathbb{Z}^{2}$ with respect to this matrix is the dyadic rationals, so by Proposition 11.3. $K_{0}\left(F_{\tau}\right)$ is isomorphic to the dyadic rationals, with order unit 1 , and with the canonical automorphism being multiplication by 2. Thus $K_{0}\left(F_{\tau}\right)$ is unitally isomorphic to $K_{0}\left(M_{2 \infty}\right)$, and $F_{\tau}$ and $M_{2 \infty}$ are unital AF-algebras, so $F_{\tau} \cong M_{2}$. The unique tracial state of $F_{\tau}$ is given by integration with respect to Lebesgue measure; cf. Lemma 10.2 Since the slopes are \pm 2 , the entropy of $\tau$ is $\ln 2$ 42, so there is a unique $\beta$-KMS state for $\beta=\ln 2$ (Theorem 10.7). By elementary operations over $\mathbb{Z}$, we can transform id $-A$ into the identity matrix, so by Corollary 11.4. $K_{0}\left(O_{\tau}\right) \cong \operatorname{coker}(\mathrm{id})=0$ and $K_{1}\left(O_{\tau}\right) \cong \operatorname{ker}(\mathrm{id})=0$. It follows that $O_{\tau} \cong O_{A} \cong O_{2}$. This information on $O_{\tau}$ can also be derived by viewing $\tau$ as a unimodal map; cf. Lemma 12.1 and Theorem 12.2.

Example 11.13. Let $\tau$ be the Markov map in Figure 1. Then the associated local homeomorphism $\sigma: X \rightarrow X$ is Markov with respect to the partition $\{I(0,1 / 3)$, $I(1 / 3,2 / 3), I(2 / 3,1)\}$, with incidence matrix

$$
A=\left(\begin{array}{lll}
0 & 1 & 1 \\
1 & 0 & 1 \\
1 & 1 & 0
\end{array}\right) .
$$

All entries of $A^{2}$ are positive, so $A$ is primitive. By Corollary [11.9] and the remarks afterwards, $F_{\tau} \cong F_{A}$ and $O_{\tau} \cong O_{A}$, and both $F_{\tau}$ and $O_{\tau}$ are simple. By elementary 
operations over $\mathbb{Z}$, we can transform id $-A$ into the diagonal matrix with entries 1 , 2,2 , so by Corollary 11.4, $K_{0}\left(O_{\tau}\right) \cong \mathbb{Z} / 2 \mathbb{Z} \oplus \mathbb{Z} / 2 \mathbb{Z}$ and $K_{1}\left(O_{\tau}\right) \cong 0$.

Since $K_{0}\left(F_{\tau}\right) \cong D G(\tau)$, and $D G(\tau)$ is described for most of the following examples in [63] and [64], in the rest of this paper we will concentrate on describing the algebras $O_{\tau}$ and their K-groups.

\section{UNIMODAL MAPS}

A map $\tau:[0,1] \rightarrow[0,1]$ is unimodal if $\tau$ is continuous, there is a single critical point $c$, and $\tau$ increases on $[0, c]$, and decreases on $[c, 1]$. If $\tau$ is unimodal and surjective, then $\tau(c)=1$, and we must have either $\tau(0)=0$ or $\tau(1)=0$ (or both). We will just consider the case where $\tau(1)=0$. (The case where $\tau(0)=0$ can be dealt with by similar techniques.)

We first analyze the case where the orbit of 0 (or equivalently, of the critical point $c)$ is periodic. Let $J_{1}=[0, c]$ and $J_{-1}=(c, 1]$, and let the itinerary of 0 be the sequence $n_{0}, n_{1}, n_{2}, \ldots$. (In other words, $\tau^{k} 0 \in J_{n_{k}}$ for $k \geq 0$.) Define $a_{k}$ to be the product $n_{0} n_{1} \cdots n_{k}$ for $k \geq 0$, and $a_{-1}=1$. With latter convention, note that $a_{k} a_{k-1}=n_{k}$ for all $k \geq 0$.

Lemma 12.1. Let $\tau:[0,1] \rightarrow[0,1]$ be a surjective unimodal map satisfying $\tau(1)=$ 0 . Assume that 0 is eventually periodic, with $0, \tau 0, \ldots, \tau^{k} 0, \ldots, \tau^{p-1} 0$ all distinct and $\tau^{p} 0=\tau^{k} 0$. With the notation for the itinerary of 0 described above, the minimal polynomial $m(t)$ of $\mathcal{L}$ on $M=\mathbb{Z}[t] I(0,1) \subset C(X, \mathbb{Z})$ is as follows:

(1) If 0 is periodic with period $p \geq 3$,

$$
m(t)=t^{p-1}-t^{p-2}-a_{0} t^{p-3}-a_{1} t^{p-4}-\cdots-a_{p-3} .
$$

(2) If 0 is periodic with period $2, m(t)=t-1$.

(3) If 0 is a fixed point of $\tau$, then $m(t)=t-2$.

(4) If 0 is eventually periodic, with $k>1$,

$$
\begin{aligned}
& m(t)=t^{p}-t^{p-1}-a_{0} t^{p-2}-a_{1} t^{p-3}-\cdots-a_{p-2} \\
& \quad-\frac{a_{k-1}}{a_{p-1}}\left(t^{k}-t^{k-1}-a_{0} t^{k-2}-a_{1} t^{k-3}-\cdots-a_{k-2}\right) .
\end{aligned}
$$

(5) If 0 is eventually periodic, with $k=1$,

$$
m(t)=t^{p}-t^{p-1}-a_{0} t^{p-2}-a_{1} t^{p-3}-\cdots-a_{p-2}-a_{p-1}(t-1) .
$$

Proof. Let $c$ be the critical point of $\tau$. Fix $j \geq 0$. If $\tau^{j} 0 \leq c$, then

$$
\mathcal{L} I\left(\tau^{j} 0,1\right)=\mathcal{L} I\left(\tau^{j} 0, c\right)+\mathcal{L} I(c, 1)=I\left(\tau^{j+1} 0,1\right)+I(0,1) .
$$

If $\tau^{j} 0>c$, then

$$
\mathcal{L} I\left(\tau^{j} 0,1\right)=I\left(0, \tau^{j+1} 0\right)=I(0,1)-I\left(\tau^{j+1} 0,1\right) .
$$

Thus in either case we have

$$
\mathcal{L} I\left(\tau^{j} 0,1\right)=n_{j} I\left(\tau^{j+1} 0,1\right)+I(0,1) .
$$

Rearranging and using $1 / n_{j}=n_{j}$ gives

$$
I\left(\tau^{j+1} 0,1\right)=n_{j} \mathcal{L} I\left(\tau^{j} 0,1\right)-n_{j} I(0,1) .
$$

By induction, for $j \geq 1, \mathcal{L}$ satisfies

$$
I\left(\tau^{j} 0,1\right)=a_{j-1}\left(\mathcal{L}^{j}-\mathcal{L}^{j-1}-a_{0} \mathcal{L}^{j-2}-a_{1} \mathcal{L}^{j-3}-\cdots-a_{j-2}\right) I(0,1) .
$$


(When $j=1$, the right side is $a_{0}(\mathcal{L}-1) I(0,1)$.) Observe that by (15), for $k \geq 0$, the subgroup generated by $\left\{I\left(\tau^{j} 0,1\right) \mid 0 \leq j \leq k\right\}$ in $C(X, \mathbb{Z})$ is contained in the subgroup generated by $\left\{\mathcal{L}^{j} I(0,1) \mid 0 \leq j \leq k\right\}$. The opposite containment follows from (15) by induction. Now we prove (11)-(5).

(11) Assume 0 is periodic with period $p \geq 3$. Then 1 is the unique pre-image of 0 , so $\tau^{p-1} 0=1$. Putting $j=p-1$ in $(15)$ gives $m(\mathcal{L}) I(0,1)=0$, where $m$ is as in (1). Here the $p-1$ vectors $\left\{I\left(\tau^{k} 0,1\right) \mid 0 \leq k \leq p-2\right\}$ are linearly independent in $C(X)$, so the vectors $\left\{\mathcal{L}^{k} I(0,1) \mid 0 \leq k \leq p-2\right\}$ are independent, and thus we conclude that (11) gives the minimal polynomial for $\mathcal{L}$.

(2) If 0 has period 2, then $\tau 0=1$ and $\tau 1=0$, so $\mathcal{L} I(0,1)=I(0,1)$. It follows that $m(t)=t-1$.

(3) If 0 is fixed, then $\mathcal{L} I(0,1)=2 I(0,1)$, so $m(t)=t-2$ is the minimal polynomial of $\mathcal{L}$.

(4) Assume that 0 is eventually periodic, with $k>1$. Then applying (15) with $j=p$ and $j=k$, we conclude that $m(\mathcal{L}) I(0,1)=0$, where $m$ is as defined in (4). Since $I(0,1)$ is a cyclic element for $\mathcal{L}$ on $M$, then $m(\mathcal{L})=0$ on $M$. We show that no polynomial of lower degree annihilates $\mathcal{L}$ on $M$. The points $\left\{\tau^{j} 0 \mid 0 \leq j \leq p-1\right\}$ are distinct, and none equals 1 , so the characteristic functions for the $p$ intervals $\left\{I\left(\tau^{j} 0,1\right) \mid 0 \leq j \leq p-1\right\}$ are linearly independent in $C(X, \mathbb{R})$. Thus the linear span $V$ of this set is $p$ dimensional. As remarked above, the linear span of the $p$ vectors $\left\{\mathcal{L}^{k} I(0,1) \mid 0 \leq k \leq p-1\right\}$ equals $V$, and thus these vectors also are linearly independent. Hence, no linear combination of $1, \mathcal{L}, \mathcal{L}^{2}, \ldots, \mathcal{L}^{p-1}$ annihilates $I(0,1)$. It follows that $m$ is the minimal polynomial of $\mathcal{L}$.

(5) Assume that 0 is eventually periodic, with $k=1$. Then combining (15) for $j=k=1$ and (15) for $j=p$ gives the desired formula for $m(t)$. That $m$ is the minimal polynomial of $\mathcal{L}$ follows in a similar way to (4).

In the statement of the following theorem, periodic points are also viewed as eventually periodic.

Theorem 12.2. Let $\tau:[0,1] \rightarrow[0,1]$ be a surjective unimodal map that satisfies $\tau(1)=0$.

(1) Assume that the critical point $c$ is eventually periodic, and let $m(t)$ be the minimal polynomial of $\mathcal{L}$ on $\mathbb{Z}[t] I(0,1)$, as described in Lemma 12.1, Let $n=|m(1)|$.

(a) If $n \neq 0$, then

$$
K_{0}\left(O_{\tau}\right) \cong \mathbb{Z} / n \mathbb{Z} \text { and } K_{1}\left(O_{\tau}\right)=0,
$$

and if in addition $\tau$ is transitive, then $O_{\tau} \cong O_{n+1}$.

(b) If $n=0$, then

$$
K_{0}\left(O_{\tau}\right) \cong \mathbb{Z} \text { and } K_{1}\left(O_{\tau}\right) \cong \mathbb{Z}
$$

(2) If 0 is not eventually periodic, then $K_{0}\left(O_{\tau}\right) \cong \mathbb{Z}$ and $K_{1}\left(O_{\tau}\right)=0$. If in addition $\tau$ is transitive, then $O_{\tau} \cong O_{\infty}$.

Proof. By Corollary [8.2, $D G(\tau)$ is generated as a $\mathbb{Z}\left[t, t^{-1}\right]$ module by $I(0, c)$ and $I(c, 1)$. Since $\mathcal{L} I(c, 1)=I(0,1)$ and $I(0, c)=I(0,1)-I(c, 1)$, then $I(0,1)$ is a cyclic element for $D G(\tau)$. If 0 is eventually periodic, the theorem follows from Proposition 11.11 and Lemma 12.1, If 0 is not eventually periodic, then $K_{0}\left(F_{\tau}\right) \cong \mathbb{Z}\left[t, t^{-1}\right]$ (as 
abelian groups), with the action of $\mathcal{L}_{*}$ given by multiplication by $t$; cf. 63. Theorem 9.1]. Now $K_{0}\left(O_{\tau}\right) \cong \mathbb{Z}$ and $K_{1}\left(O_{\tau}\right) \cong 0$ follow from Proposition 9.4 These are the same K-groups as those for $O_{\infty}$. If $\tau$ is transitive, since it is continuous, it can not be essentially injective. Thus by Theorem $9.5, O_{\tau}$ meets the conditions needed to apply the Kirchberg/Phillips classification results (Theorem 9.6), so $O_{\tau} \cong O_{\infty}$.

Given $1<s<2$, we define the restricted tent map $T_{s}$ by

$$
T_{s}(x)= \begin{cases}1+s(x-c) & \text { if } x \leq c, \\ 1-s(x-c) & \text { if } x>c,\end{cases}
$$

where $c=1-1 / s$. (This is the usual symmetric tent map $\tau$ on $[0,1]$ with slopes $\pm s$, restricted to the interval $\left[\tau^{2}(1 / 2), \tau(1 / 2)\right]$, which is the interval of most interest for the dynamics. Then the map has been rescaled so that its domain is $[0,1]$. See Figure 1,) Note that $T_{s}(c)=1$ and $T_{s}(1)=0$.

Example 12.3. Let $\tau=T_{s}$ be the restricted tent map, with $\sqrt{2}<s<2$, and assume that the orbit of the critical point is not eventually periodic. (For example, this occurs if $s=3 / 2$, or if $s$ is transcendental.) By [25] or [64, Lemma 8.1], $\tau$ is topologically exact. By Theorem 12.2, $O_{\tau} \cong O_{\infty}$. Thus all such tent maps give isomorphic $\mathrm{C}^{*}$-algebras $O_{\tau}$. However, $O_{\tau}$ comes equipped with an action of $\mathbb{T}$ (or of $\mathbb{R}$ ) (see the discussion preceding Theorem 10.7), and these actions give different KMS states. In fact, by Theorem 10.7 since $\ln s$ is the entropy of $\tau=T_{s}$, there will be a unique $\beta$-KMS state for $\beta=\ln s$. Furthermore, the range of the unique state on the dimension group of the AF-algebras $F_{\tau}$ will be $\mathbb{Z}\left[s, s^{-1}\right]$ (64, Prop. 8.2]). As $s$ varies, many of the algebras $F_{\tau}$ for $\tau=T_{s}$ will be non-isomorphic, even though the associated algebras $O_{\tau}$ will all be isomorphic to $O_{\infty}$.

Example 12.4. Let $\tau_{0}(x)=k x(1-x)$, with $c$ the unique critical point, and choose $k \approx 3.68$ so that $p=\tau_{0}^{3}(c)$ is fixed. Let $\tau$ denote $\left.\tau_{0}\right|_{J}$, where $J=\left[\tau_{0}^{2}(c), \tau_{0}(c)\right]$, rescaled so that the domain of the restricted map is [0,1]. Applying Lemma 12.1(5) (with $k=1$ and $p=2$, since $\tau^{3} c=\tau 0$ is fixed) we conclude that the minimal polynomial of $\mathcal{L}$ on $\mathbb{Z}[t] I(0,1)$ is $m(t)=t^{2}-2$. By Theorem 12.2, $K_{0}\left(O_{\tau}\right)=0$ and $K_{1}\left(O_{\tau}\right)=0$. By [63, Example 9.5], $\tau$ is conjugate to $T_{\sqrt{2}}$, which is transitive [64, Lemma 8.1], so by Theorem [12.2. $O_{\tau} \cong O_{2}$.

\section{Multimodal maps}

By a multimodal map we mean a continuous piecewise monotonic map. In this section, we compute the $K$-groups of $O_{\tau}$ for some multimodal maps.

Example 13.1. Let $\tau: I \rightarrow I$ be piecewise monotonic, continuous, and surjective, with $0=a_{0}<a_{1}<\cdots<a_{q}=1$ being the associated partition. Assume that the orbits of $a_{1}, \ldots, a_{q-1}$ are disjoint and infinite, and $\tau(\{0,1\}) \cap\{0,1\}=\emptyset$. Then by Corollary 7.10 and 63 , Prop. 10.6], $K_{0}\left(F_{\tau}\right) \cong D G(\tau) \cong\left(\mathbb{Z}\left[t, t^{-1}\right]\right)^{q-1}$, with the action of $\mathcal{L}_{*}$ on $D G(\tau)$ given by multiplication by $t$. By Proposition 9.4, $K_{0}\left(O_{\tau}\right) \cong$ $\operatorname{coker}(1-t) \cong \mathbb{Z}^{q-1}$ and $K_{1}\left(O_{\tau}\right) \cong \operatorname{ker}(1-t) \cong 0$.

Example 13.2. Let $\tau: I \rightarrow I$ be continuous, piecewise linear, and topologically mixing, with slopes $\pm s$, with associated partition $a_{0}<a_{1}<\cdots<a_{n}$. Assume

(1) $s$ is transcendental, 
(2) the lengths of the intervals $\left[a_{0}, a_{1}\right], \ldots,\left[a_{n-2}, a_{n-1}\right]$ are independent over $\mathbb{Z}\left[s, s^{-1}\right]$,

(3) $\tau(\{0,1\}) \cap\{0,1\}=\emptyset$.

Then by [64, Prop. 9.2], $D G(\tau)$ is order isomorphic to $\sum_{i=1}^{n-1} \mathbb{Z}\left[s, s^{-1}\right]\left(a_{i}-a_{i-1}\right)$, with the action of $\mathcal{L}_{*}$ given by multiplication by $s$. As in Example 13.1, we find $K_{0}\left(O_{\tau}\right) \cong \mathbb{Z}^{n-1}$, and $K_{1}\left(O_{\tau}\right) \cong 0$.

Observe that for the maps in Examples 13.1 and 13.2, $K_{1}\left(O_{\tau}\right)$ is not the torsionfree part of $K_{0}\left(O_{\tau}\right)$, so $O_{\tau}$ will not be a Cuntz-Krieger algebra.

\section{INTERVAL EXCHANGE MAPS}

Definition 14.1. A piecewise monotonic map $\tau:[0,1) \rightarrow[0,1)$ is a generalized interval exchange map if $\tau$ is increasing on each interval of monotonicity, is bijective, and is right continuous at all points. We will usually identify $\tau$ with its extension to a map from $[0,1]$ into $[0,1]$, defined to be left continuous at 1 .

Note that if $0=a_{0}<a_{1}<\cdots<a_{n}=1$ is the partition associated with a generalized interval exchange map $\tau$, then $\tau$ maps the intervals $\left[a_{i-1}, a_{i}\right)$ to new intervals which partition $[0,1)$. If a generalized interval exchange map is linear with slope 1 on each interval of monotonicity, it is called an interval exchange map; in this case $\tau$ maps each interval $\left[a_{i-1}, a_{i}\right)$ to an interval of the same length.

For a generalized interval exchange map, since $\tau:[0,1) \rightarrow[0,1)$ is bijective, the associated local homeomorphism $\sigma: X \rightarrow X$ will be a homeomorphism. Then, as we observed when $C^{*}(X, \sigma)$ was defined, $C^{*}(X, \sigma)$ will be isomorphic to the transformation group $\mathrm{C}^{*}$-algebra $C(X) \times_{\psi} \mathbb{Z}$, where the action of $\mathbb{Z}$ is given by the *-automorphism $\psi$ defined by $\psi(f)=f \circ \sigma^{-1}$. If $\tau$ is an interval exchange map, then our construction of the homeomorphism $\sigma: X \rightarrow X$ is the same as that of Putnam [55]. Thus we have the following.

Proposition 14.2. If $\tau: I \rightarrow I$ is an interval exchange map, then $O_{\tau}$ coincides with the transformation group $C^{*}$-algebra associated with interval exchange maps by Putnam in [55].

When we have referred to the generalized orbit of a point with respect to a piecewise monotonic map with some discontinuities, we have previously used the orbit with respect to the multivalued map $\widehat{\tau}$. In the next proposition, we need to refer to the orbit with respect to $\tau$ itself, and we will call this the $\tau$-orbit to avoid confusion with our earlier usage. If a set $B$ has the property that its points have orbits that are infinite and disjoint, we refer to this property as the IDOC for $B$.

In [63, Corollary 11.8], the dimension group $D G(\tau)$ was described for generalized interval exchange maps in which the orbits of the partition endpoints $a_{1}, a_{2}, \ldots, a_{n-1}$ satisfy the IDOC. Since the associated local homeomorphism $\sigma$ is a homeomorphism, then as a group, $D G(\tau)=C(X, \mathbb{Z})$. As a $\mathbb{Z}\left[t, t^{-1}\right]$ module, $D G(\tau) \cong \mathbb{Z}\left[t, t^{-1}\right]^{n-1} \oplus \mathbb{Z}$, where the action of $\mathcal{L}_{*}$ is multiplication by $t$ on the first summand, and trivial on the $\mathbb{Z}$ summand. We now apply this to compute the K-groups of $O_{\tau}$. For interval exchange maps, Proposition 14.3 is due to Putnam [55].

Proposition 14.3. Let $\tau: I \rightarrow I$ be a generalized interval exchange map, with associated partition $C=\left\{a_{0}, a_{1}, \ldots, a_{n}\right\}$. If the $\tau$-orbits of $a_{1}, a_{2}, \ldots, a_{n-1}$ are infinite and disjoint, then $K_{0}\left(O_{\tau}\right) \cong \mathbb{Z}^{n}$ and $K_{1}\left(O_{\tau}\right) \cong \mathbb{Z}$. 
Proof. Let $M=\left(\mathbb{Z}\left[t, t^{-1}\right]\right)^{n-1}$. By the result from [63, Corollary 11.8] described above, $C(X, \mathbb{Z})=D G(\tau) \cong M \oplus \mathbb{Z}$, and $\left(\right.$ id $\left.-\mathcal{L}_{*}\right) D G(\tau)=(1-t) M \oplus 0$. Thus $C(X, \mathbb{Z}) /(\mathrm{id}-\mathcal{L}) C(X, \mathbb{Z}) \cong M /(1-t) M \oplus \mathbb{Z}$.

Let $\psi: M \rightarrow \mathbb{Z}^{n-1}$ be the map $\psi\left(p_{1}, \ldots, p_{n-1}\right)=\left(p_{1}(1), \ldots, p_{n-1}(1)\right)$. Then $\psi$ is a homomorphism from $M$ onto $\mathbb{Z}^{n-1}$. The kernel consists of those $\left(p_{1}, \ldots, p_{n-1}\right) \in$ $M$ such that $p_{i}(1)=0$ for each $i$. If $\left(p_{1}, \ldots, p_{n-1}\right) \in \operatorname{ker} \psi$, for each $i$ we can write $p_{i}(t)=(1-t) q_{i}(t)$ for $q_{i} \in \mathbb{Z}\left[t, t^{-1}\right]$, so $\left(p_{1}, \ldots, p_{n-1}\right) \in(1-t) M$. Conversely, each element in $(1-t) M$ is in the kernel of the homomorphism. Thus $M /(1-t) M \cong$ $\mathbb{Z}^{n-1}$, and so $K_{0}\left(O_{\tau}\right) \cong \mathbb{Z}^{n-1} \oplus \mathbb{Z} \cong \mathbb{Z}^{n}$. Finally, $K_{1}\left(F_{\tau}\right) \cong \operatorname{ker}(1-t) \cong 0 \oplus \mathbb{Z} \cong$ $\mathbb{Z}$.

\section{5. $\beta$-TRANSFORMATIONS}

If $\beta>1$, the $\beta$-transformation on $\left[0,1\right.$ ) is the map $\tau_{\beta}: x \mapsto \beta x \bmod 1$. (See Figure 1.) We extend $\tau_{\beta}$ to $[0,1]$ by defining $\tau_{\beta}(1)=\lim _{x \rightarrow 1^{-}} \tau_{\beta}(x)$. Then $\tau_{\beta}$ is piecewise monotonic, and the associated partition is $\{0,1 / \beta, 2 / \beta, \ldots, n / \beta, 1\}$, where $n=[\beta]$ is the greatest integer $\leq \beta$.

Katayama, Matsumoto, and Watatani [27] have associated $\mathrm{C}^{*}$-algebras $F_{\beta}^{\infty}$ and $O_{\beta}$ with the $\beta$-shift. In this section we will show that when $\tau$ is a $\beta$-transformation, the algebras $O_{\tau}$ and $O_{\beta}$ are isomorphic. If 1 is eventually periodic, then $F_{\tau} \cong F_{\beta}^{\infty}$.

The $\beta$-transformation is always topologically exact ([64, Lemma 10.1]). We need the following result describing the minimal polynomial of $\mathcal{L}$ on $\mathbb{Z}[t] I(0,1)$. If $x \in I$, and $n$ is the greatest integer $\leq \beta$, we give the intervals $[0,1 / \beta),[1 / \beta, 2 / \beta), \ldots$, $[(n-1) / \beta, n / \beta),[n / \beta, 1]$ the labels $0,1, \ldots, n$, and define the itinerary of $x \in I$ to be $n_{0} n_{1} n_{2} \ldots$, where $\tau^{k} x$ is in the interval with label $n_{k}$. The calculation of the minimal polynomial in the following result is similar to our previous calculation for unimodal maps (cf. Lemma 12.1) and so is left to the reader.

Lemma 15.1 (64, Lemma 10.2]). Let $\tau$ be the $\beta$-transformation, and $\sigma: X \rightarrow$ $X$ the associated local homeomorphism. Assume 1 is eventually periodic, with $1, \tau 1, \ldots, \tau^{p-1} 1$ distinct, and with $\tau^{p} 1=\tau^{k} 1$ for some $k<p$. Let the itinerary of 1 be $n_{0} n_{1} n_{2} \cdots n_{k} \cdots n_{p} \cdots$, and let $M=\mathbb{Z}[t] I(0,1)$. Then $M$ is order isomorphic to $\mathbb{Z}^{p}$. Let $m(t)$ be the minimal polynomial for $\mathcal{L}$ restricted to $M$.

(1) If $\tau 1=1$, then $m(t)=t-n_{0}$.

(2) If $\tau 1 \neq 1$ and $\tau^{p} 1 \neq 0$, then

$$
m(t)=t^{p}-n_{0} t^{p-1}-n_{1} t^{p-2}-\cdots-n_{p-1}-\left(t^{k}-n_{0} t^{k-1}-\cdots-n_{k-1}\right) .
$$

(3) If $\tau^{p} 1=0$, then

$$
m(t)=t^{p-1}-n_{0} t^{p-2}-n_{1} t^{p-3}-\cdots-n_{p-2} .
$$

As discussed before Theorem 10.7 there is a natural action of $\mathbb{R}$ on $O_{\tau}$, so it makes sense to speak of KMS states on $O_{\tau}$. In the following, we use the notation of Lemma 15.1.

Proposition 15.2. Let $\tau$ be the $\beta$-transformation.

(1) If 1 is eventually periodic, let

$$
n=|m(1)|= \begin{cases}n_{0}-1 & \text { if } \tau 1=1, \\ \sum_{i=k}^{p-1} n_{i} & \text { if } \tau 1 \neq 1 \text { and } \tau^{p} 1 \neq 0, \\ \left(\sum_{i=0}^{p-2} n_{i}\right)-1 & \text { if } \tau^{p} 1=0 .\end{cases}
$$


Then $n \neq 0$, and $K_{0}\left(O_{\tau}\right) \cong \mathbb{Z} / n \mathbb{Z}$ and $K_{1}\left(O_{\tau}\right)=0$. Furthermore, $O_{\tau} \cong$ $O_{n+1}$.

(2) If 1 is not eventually periodic, then $K_{0}\left(O_{\tau}\right) \cong \mathbb{Z}$ and $K_{1}\left(O_{\tau}\right)=0$, and $O_{\tau} \cong O_{\infty}$.

(3) $F_{\tau}$ is a simple AF algebra with a unique trace given by Lebesgue measure.

(4) The entropy of $\tau$ is $\ln \beta$, and there is a unique $(\ln \beta)-K M S$ state for $O_{\tau}$.

Proof. Assume 1 is eventually periodic. To show $n \neq 0$, we consider three cases. If $\tau 1=1$, then $n_{0}=\beta \geq 2$, so $m(1)=n_{0}-1 \neq 0$. If $\tau 1 \neq 1$, and $\tau^{p} 1 \neq 0$, suppose that $n=|m(1)|=0$. Then $n_{k}=n_{k+1}=\cdots=n_{p-1}=0$. Since $\sigma^{p} 1=\sigma^{k} 1$, then $n_{j}=0$ for all $j \geq k$. It follows that the orbit of 1 stays in the interval $[0,1 / \beta)$ forever. This is possible only if the orbit of 1 lands on 0 , which contradicts $\tau^{p} 1 \neq 0$. If $\tau^{p} 1=0$, then $\tau^{p-1} 1=0$, and $\tau^{p-2} 1=n_{p-2} / \beta$, where $n_{p-2} \geq 1$. Since $n_{0} \geq 1$, then $n \geq 1$ follows. Thus we have shown that $n \neq 0$. Now (1) follows from Proposition 11.11.

If 1 is not eventually periodic, then $K_{0}\left(F_{\tau}\right) \cong D G(\tau) \cong \mathbb{Z}\left[t, t^{-1}\right]$, with the action of $\mathcal{L}_{*}$ given by multiplication by $t$ (cf. [64, Prop. 10.5]), so the statements about $K_{0}\left(O_{\tau}\right)$ and $K_{1}\left(O_{\tau}\right)$ in (2) follow from Proposition 9.4. Since $\tau$ is topologically exact, we can apply Theorem 9.5 and the Kirchberg-Phillips classification results (Theorem 9.6) to conclude that $O_{\tau} \cong O_{\infty}$.

Since $\tau$ is exact, then (3) follows from Corollary 10.6 The fact that the entropy of $\tau$ is $\ln \beta$ can be found in [64, Prop. 3.7]. The statement about KMS states follows from Theorem 10.7 .

If $x \in[0,1]$, then the $\beta$-expansion of $x$ is $x=\frac{n_{0}}{\beta}+\frac{n_{1}}{\beta^{2}}+\cdots$, where $n_{0}$ is the greatest integer in $\beta x$, and for each $k \geq 1, n_{k}$ is the greatest integer in $\beta^{k+1}\left(x-\sum_{i=0}^{k-1} \frac{n_{i}}{\beta^{i+1}}\right)$. If $\beta \notin \mathbb{N}$, the itinerary of 1 will be the sequence of coefficients $\left(n_{1} n_{2} \cdots\right)$ in the $\beta$-expansion of 1. (If $\beta=n \in \mathbb{N}$, the itinerary of 1 will be $(n-1)(n-1) \cdots$, while the $\beta$-expansion of 1 will be $n 000 \cdots$.) The $\beta$-expansion of 1 will be finite iff either $\tau 1=1$ or the orbit of 1 lands on 0 . Since $\tau$ is topologically exact, itineraries separate points, so the $\beta$-expansion of 1 will eventually be periodic iff 1 is eventually periodic.

Corollary 15.3. If $\tau$ is the $\beta$-transformation, then the $C^{*}$-algebras $O_{\tau}$ and $O_{\beta}$ are isomorphic. If 1 is eventually periodic, then $F_{\tau} \cong F_{\beta}^{\infty}$.

Proof. From Proposition 15.2, $O_{\tau} \cong O_{n+1}$ if 1 is not eventually periodic, and $O_{\tau} \cong O_{\infty}$ otherwise. Here $n$ is described in terms of the itinerary of 1 . In [27, Cor. 4.13], it also is shown that $O_{\beta} \cong O_{n+1}$ if the $\beta$-expansion of 1 is eventually periodic, and $O_{\beta} \cong O_{\infty}$ otherwise. A formula for $n$ is given in terms of the $\beta$-expansion of 1 ; this formula gives the same value for $n$ as in Proposition 15.2. Thus $O_{\tau} \cong O_{\beta}$ in either case.

If 1 is eventually periodic, it is shown in [64, Prop. 10.6] that $D G(\tau)$ and $K_{0}\left(F_{\beta}^{\infty}\right)$ are unitally order isomorphic. Thus $F_{\tau}$ and $F_{\beta}^{\infty}$ are unital AF-algebras with unitally order isomorphic $K_{0}$ groups, so are isomorphic by Elliott's classification of AFalgebras [18.

\section{REFERENCES}

[1] C. Anantharaman-Delaroche, Purely infinite $C^{*}$-algebras arising from dynamical systems. Bull. Soc. Math. France 125 (1997), no. 2, 199-225. MR1478030(99i:46051) 
[2] V. Arzumanian and J. Renault, Examples of pseudogroups and their $C^{*}$-algebras. Operator algebras and quantum field theory (Rome, 1996), 93-104, Internat. Press, Cambridge, MA, 1997. MR1491110 (99a:46101)

[3] V. A. Arzumanian and A. M. Vershik, Star algebras associated with endomorphisms. Operator algebras and group representations, Vol. I (Neptun, 1980), 17-27, Monogr. Stud. Math., 17, Pitman, Boston, MA, 1984. MR0731760 (85k:46063)

[4] B. Blackadar, Symmetries of the CAR algebra. Ann. of Math. (2) 131 (1990), no. 3, 589-623. MR.1053492(91i:46084)

[5] B. Brenken, C*-algebras associated with topological relations, J. Ramanujan Math. Soc. 19 (2004), no. 1, 35-55. MR2054608 (2004m:46134)

[6] L. S. Block and W. A. Coppel, Dynamics in one dimension. Lecture Notes in Mathematics, 1513. Springer-Verlag, Berlin, 1992. MR1176513 (93g:58091)

[7] M. Boyle, D. Fiebig, and U. Fiebig, A dimension group for local homeomorphisms and endomorphisms of one-sided shifts of finite type. J. Reine Angew. Math. 487 (1997), 27-59. MR 1454258 (98i:54020)

[8] P. Collet and J. Eckmann, Iterated maps on the interval as dynamical systems, Progress in Physics 1, Birkhäuser, Boston, 1980. MR0613981 (82j:58078)

[9] J. Cuntz, A class of $C^{*}$-algebras and topological Markov chains. II. Reducible chains and the Ext-functor for $C^{*}$-algebras. Invent. Math. 63 (1981), no. 1, 25-40. MR0608527(82f:46073b)

[10] J. Cuntz and W. Krieger, A class of $\mathrm{C}^{*}$-algebras and topological Markov chains. Invent. Math. 56 (1980), no. 3, 251-268. MR0561974 (82f:46073a)

[11] K. Davidson, $C^{*}$-algebras by example. Fields Institute Monographs, 6. American Mathematical Society, Providence, RI, 1996. MR 1402012 (97i:46095)

[12] V. Deaconu, Groupoids associated with endomorphisms. Trans. Amer. Math. Soc. 347 (1995), no. 5, 1779-1786. MR.1233967 (95h:46104)

[13] V. Deaconu and P. Muhly, $\mathrm{C}^{*}$-algebras associated with branched coverings. Proc. Amer. Math. Soc. 129 (2001), no. 4, 1077-1086 (electronic). MR.1814145(2002a:46075)

[14] V. Deaconu and F. Shultz, $\mathrm{C}^{*}$-algebras associated with interval maps, 40 pages, arXiv:math. $\mathrm{OA} / 0405469$

[15] M. Denker, and M. Urbański, On the existence of conformal measures. Trans. Amer. Math. Soc. 328 (1991), no. 2, 563-587. MR.1014246 (92k:58155)

[16] E. Effros, Dimensions and $C^{*}$-algebras. CBMS Regional Conference Series in Mathematics, 46. Conference Board of the Mathematical Sciences, Washington, D.C., 1981. MR0623762 (84k:46042)

[17] E. G. Effros, D. E. Handelman, and C. L. Shen, Dimension groups and their affine representations. Amer. J. Math. 102 (1980), no. 2, 385-407. MR.0564479 (83g:46061)

[18] G. Elliott, On the classification of inductive limits of sequences of semisimple finitedimensional algebras. J. Algebra 38 (1976), no. 1, 29-44. MR0397420 (53:1279)

[19] G. Elliott, On the classification of C*-algebras of real rank zero, J. Reine Angew. Math. 443 (1993), 179-219. MR1241132 (94i:46074)

[20] R. Exel, personal communication.

[21] R. Exel and A. Vershik, C*-algebras of irreversible dynamical systems. Canad. J. Math. 58 (2006), no. 1, 39-63. MR2195591

[22] T. Giordano, I. Putnam, and C. Skau, Topological orbit equivalence and C*-crossed products. J. Reine Angew. Math. 469 (1995), 51-111. MR1363826 (97g:46085)

[23] K. R. Goodearl, Partially ordered abelian groups with interpolation. Mathematical Surveys and Monographs, 20. American Mathematical Society, Providence, RI, 1986. MR0845783 (88f:06013)

[24] F. Hofbauer, The structure of piecewise monotonic transformations. Ergodic Theory Dynamical Systems 1 (1981), no. 2, 159-178. MR0661817(83k:58065)

[25] L. Jonker and D. Rand, Bifurcations in one dimension. I. The nonwandering set. Invent. Math. 62 (1981), no. 3, 347-365. MR0604832(83d:58057a)

[26] T. Kajiwara and Y. Watatani, $\mathrm{C}^{*}$-algebras associated with complex dynamical systems. Indiana Univ. Math. J. 54 (2005), no. 3, 755-778. MR2151233 (2006b:46074)

[27] Y. Katayama, K. Matsumoto, and Y. Watatani, Simple $C^{*}$-algebras arising from $\beta$-expansion of real numbers. Ergodic Theory Dynam. Systems 18 (1998), no. 4, 937-962. MR1645334 (99m:46136) 
[28] T. Katsura, A class of $\mathrm{C}^{*}$-algebras generalizing both graph algebras and homeomorphism C*-algebras I, fundamental results. Trans. Amer. Math. Soc. 356 (2004), no. 11, 4287-4322. MR2067120 (2005b:46119)

[29] T. Katsura, On C*-algebras associated with C*-correspondences, J. Funct. Anal. 217 (2004), no. 2, 366-401. MR2102572 (2005e:46099)

[30] M. Keane, Interval exchange transformations. Math. Z. 141 (1975), 25-31. MR0357739 (50:10207)

[31] M. Keane, Non-ergodic interval exchange transformations. Israel J. Math. 26 (1977), no. 2, 188-196. MR0435353 (55:8313)

[32] H. Keynes and D. Newton, A "minimal", non-uniquely ergodic interval exchange transformation. Math. Z. 148 (1976), no. 2, 101-105. MR0409766 (53:13518)

[33] E. Kirchberg, The classification of purely infinite $\mathrm{C}^{*}$-algebras using Kasparov's theory, to appear in the Fields Institute Communication series.

[34] W. Krieger, On a dimension for a class of homeomorphism groups. Math. Ann. 252 (1979/80), no. 2, 87-95. MR0593623 (82b:46083)

[35] W. Krieger, On dimension functions and topological Markov chains. Invent. Math. 56 (1980), no. 3, 239-250. MR0561973 (81m:28018)

[36] A. Kumjian, Preliminary algebras arising from local homeomorphisms. Math. Scand. 52 (1983), no. 2, 269-278. MR0702958 (85b:46078)

[37] A. Kumjian, Fell bundles over groupoids. Proc. Amer. Math. Soc. 126 (1998), no. 4, 11151125. MR1443836 (98i:46055)

[38] A. Kumjian, D. Pask, and I. Raeburn, Cuntz-Krieger algebras of directed graphs. Pacific J. Math. 184 (1998), no. 1, 161-174. MR1626528 (99i:46049)

[39] A. Kumjian and J. Renault, KMS states on $\mathrm{C}^{*}$-algebras associated to expansive maps, Proc. Amer. Math. Soc. 134 (2006), no. 7, 2067-2078. MR2215776

[40] N. Martins, R. Severino, and J. S. Ramos, $K$-theory for Cuntz-Krieger algebras arising from real quadratic maps. Int. J. Math. Math. Sci. 2003, no. 34, 2139-2146. MR.1991959 (2004g:46085)

[41] P. S. Muhly, J. N. Renault, and D. P. Williams, Equivalence and isomorphism for groupoid $C^{*}$-algebras. J. Operator Theory 17 (1987), no. 1, 3-22. MR0873460 (88h:46123)

[42] M. Misiurewicz and W. Szlenk, Entropy of piecewise monotone mappings. Studia Math. 67 (1980), no. 1, 45-63. MR0579440 (82a:58030)

[43] D. Lind and B. Marcus, An introduction to symbolic dynamics and coding. Cambridge University Press, Cambridge, 1995. MR1369092 (97a:58050)

[44] W. de Melo and S. van Strien, One-dimensional dynamics. Ergebnisse der Mathematik und ihrer Grenzgebiete (3) [Results in Mathematics and Related Areas (3)], 25. Springer-Verlag, Berlin, 1993. MR1239171 (95a:58035)

[45] M. Newman, Integral Matrices, Pure and Applied Mathematics, Vol. 45. Academic Press, New York, London, 1972. MR0340283 (49:5038)

[46] W. Parry, Symbolic dynamics and transformations of the unit interval. Trans. Amer. Math. Soc. 122 (1966), 368-378. MR0197683 (33:5846)

[47] W. Parry and S. Tuncel, Classification problems in ergodic theory. London Mathematical Society Lecture Note Series, 67. Statistics: Textbooks and Monographs, 41. Cambridge University Press, Cambridge, New York, 1982. MR0666871 (84g:28024)

[48] W. L. Paschke, The crossed product of a $C^{*}$-algebra by an endomorphism. Proc. Amer. Math. Soc. 80 (1980), no. 1, 113-118. MR.0574518 (81m:46081)

[49] W. L. Paschke, $K$-theory for actions of the circle group on $C^{*}$-algebras. J. Operator Theory 6 (1981), no. 1, 125-133. MR0637006 (82m:46074)

[50] N. C. Phillips, A classification theorem for nuclear purely infinite simple $C^{*}$-algebras. Doc. Math. 5 (2000), 49-114 (electronic). MR.1745197(2001d:46086b)

[51] M. Pimsner, A class of $\mathrm{C}^{*}$-algebras generalizing both Cuntz-Krieger algebras and crossed products by $\mathbb{Z}$. Free probability theory (Waterloo, ON, 1995), 189-212, Fields Inst. Commun., 12, Amer. Math. Soc., Providence, RI, 1997. MR1426840 (97k:46069)

[52] M. Pimsner and D. Voiculescu, $K$-groups of reduced crossed products by free groups. $J$. Operator Theory 8 (1982), no. 1, 131-156. MR0670181 (84d:46092)

[53] Y. T. Poon, A $K$-theoretic invariant for dynamical systems, Trans. Amer. Math. Soc. 311 (1989), 515-533. MR0978367 (90c:46091) 
[54] C. Preston, Iterates of piecewise monotone mappings on an interval. Lecture Notes in Mathematics, 1347. Springer-Verlag, Berlin, 1988. MR0969131 (89m:58109)

[55] I. Putnam, The $\mathrm{C}^{*}$-algebras associated with minimal homeomorphisms of the Cantor set, Pacific J. Math. 136 (1989), no. 2, 329-353. MR0978619 (90a:46184)

[56] I. Putnam, $\mathrm{C}^{*}$-algebras arising from interval exchange transformations. J. Operator Theory 27 (1992), no. 2, 231-250. MR1249645 (95a:46090)

[57] I. Putnam, K. Schmidt, and C. Skau, C*-algebras associated with Denjoy homeomorphisms of the circle. J. Operator Theory 16 (1986), no. 1, 99-126. MR0847334 (87j:46126)

[58] J. Renault, A groupoid approach to $C^{*}$-algebras. Lecture Notes in Mathematics, 793. Springer, Berlin, 1980. MR0584266 (82h:46075)

[59] J. Renault, Cuntz-like Algebras, Operator theoretical methods (Timişoara, 1998), Theta Found., Bucharest, 2000, pp. 371-386. MR.1770333 (2001g:46130)

[60] J. Renault, The Radon-Nikodym problem for approximately proper equivalence relations, Ergodic Theory Dynam. Systems 25 (2005), no. 5, 1643-1672. MR2173437 (2006h:46065)

[61] M. Rørdam, F. Larsen, and N. J. Lausten, An Introduction to K-theory for C*-algebras. London Mathematical Society Student Texts 49, Cambridge University Press, Cambridge, 2000. MR1783408 (2001g:46001)

[62] D. Ruelle, Dynamical zeta functions for piecewise monotone maps of the interval. CRM Monograph Series, 4. American Mathematical Society, Providence, RI, 1994. MR1274046 (95m:58101)

[63] F. Shultz, Dimension groups for interval maps, New York J. Math. 11 (2005), 477-517. MR2188252 (2006g:37062)

[64] F. Shultz, Dimension groups for interval maps II: The transitive case, Ergodic Theory Dynamical Systems, to appear. arXiv:math.DS/0405467.

[65] J. Spielberg, Cuntz-Krieger algebras associated with Fuchsian groups. Ergodic Theory Dynam. Systems 13 (1993), no. 3, 581-595. MR1245830 (94k:46143)

[66] P. Walters, Equilibrium states for $\beta$-transformations and related transformations, Math. $Z$. 159 (1978), 65-88. MR0466492(57:6370)

[67] P. Walters, An introduction to ergodic theory. Graduate Texts in Mathematics, 79. SpringerVerlag, New York, Berlin, 1982. MR0648108 (84e:28017)

Department of Mathematics, University of Nevada, Reno, Nevada 89557

E-mail address: vdeaconu@unr.edu

Department of Mathematics, Wellesley College, Wellesley, Massachusetts 02481

E-mail address: fshultz@wellesley.edu 